\title{
THE INFLUENCE OF SUPERNOVA REMNANTS ON THE INTERSTELLAR MEDIUM IN THE LARGE MAGELLANIC CLOUD SEEN AT 20-600 $\mu \mathrm{m}$ WAVELENGTHS
}

\author{
Maša Lakićević ${ }^{1}$, Jacco Th. van Loon $^{1}$, Margaret MeiXner ${ }^{2,3}$, Karl Gordon $^{2,4}$, Caroline Bot $^{5}$, Julia Roman-Duval ${ }^{2}$, \\ Brian Babler $^{6}$, Alberto Bolatto ${ }^{7}$, Chad Engelbracht ${ }^{8}$, Miroslav Filipović $^{9}$, Sacha Hony $^{10}$, Remy Indebetouw $^{11,12}$, \\ Karl Misselt $^{8}$, Edward Montiel $^{8,13}$, K. Okumura ${ }^{10}$, Pasquale Panuzzo ${ }^{10,14}$, Ferdinando Patat ${ }^{15}$, Marc Sauvage ${ }^{10}$, \\ Jonathan Seale ${ }^{2,16}$, George Sonneborn ${ }^{17}$, Tea Temim ${ }^{17,18}$, Dejan Urošević ${ }^{19,20}$, and Giovanna Zanardo ${ }^{21}$ \\ ${ }^{1}$ Lennard-Jones Laboratories, Keele University, Staffordshire ST5 5BG, UK; m.lakicevic@ keele.ac.uk \\ ${ }^{2}$ Space Telescope Science Institute, 3700 San Martin Dr., Baltimore, MD 21218, USA \\ ${ }^{3}$ Department of Physics and Astronomy, Johns Hopkins University, 366 Bloomberg Center, 3400 N. Charles Street, Baltimore, MD 21218, USA \\ ${ }^{4}$ Sterrenkundig Observatorium, Universiteit Gent, Gent, Belgium \\ ${ }^{5}$ Observatoire astronomique de Strasbourg, Université de Strasbourg, CNRS, UMR 7550, 11 rue de l'université, F-67000 Strasbourg, France \\ ${ }^{6}$ Department of Astronomy, 475 north Charter St., University of Wisconsin, Madison, WI 53706, USA \\ ${ }^{7}$ Laboratory of Millimeter Astronomy, University of Maryland, College Park, MD 29742, USA \\ ${ }^{8}$ Steward Observatory, University of Arizona, 933 North Cherry Ave., Tucson, AZ 85721, USA \\ ${ }^{9}$ University of Western Sydney, Locked Bag 1797, Penrith South DC, NSW 1797, Australia \\ ${ }^{10}$ CEA, Laboratoire AIM, Irfu/SAp, Orme des Merisiers, F-91191 Gif-sur-Yvette, France \\ ${ }^{11}$ Department of Astronomy, University of Virginia, P.O. Box 400325, Charlottesville, VA 22903, USA \\ 12 National Radio Astronomy Observatory, 520 Edgemont Road, Charlottesville, VA 22903, USA \\ ${ }^{13}$ Louisiana State University, Department of Physics \& Astronomy, 233-A Nicholson Hall, Tower Dr., Baton Rouge, LA 70803, USA \\ ${ }^{14}$ CNRS, Observatoire de Paris - Lab. GEPI, Bat. 11, 5, place Jules Janssen, F-92195 Meudon CEDEX, France \\ ${ }^{15}$ European Organization for Astronomical Research in the Southern Hemisphere (ESO), Karl-Schwarzschild-Straße 2, \\ D-85748 Garching bei München, Germany \\ 16 The Johns Hopkins University, Department of Physics and Astronomy, 366 Bloomberg Center, \\ 3400 N. Charles Street, Baltimore, MD 21218, USA \\ 17 NASA Goddard Space Flight Center, Code 665, Greenbelt, MD 20771, USA \\ ${ }^{18}$ CRESST, University of Maryland, College Park, MD 20742, USA \\ ${ }^{19}$ Department of Astronomy, Faculty of Mathematics, University of Belgrade, Studentski trg 16, 11000 Belgrade, Serbia \\ ${ }^{20}$ Isaac Newton Institute of Chile, Yugoslavia Branch, Yugoslavia \\ ${ }^{21}$ International Centre for Radio Astronomy Research (ICRAR), M468, University of Western Australia, Crawley, WA 6009, Australia \\ Received 2014 August 26; accepted 2014 October 20; published 2015 January 14
}

\begin{abstract}
We present the analysis of supernova remnants (SNRs) in the Large Magellanic Cloud (LMC) and their influence on the environment at far-infrared (FIR) and submillimeter wavelengths. We use new observations obtained with the Herschel Space Observatory and archival data obtained with the Spitzer Space Telescope, to make the first FIR atlas of these objects. The SNRs are not clearly discernible at FIR wavelengths; however, their influence becomes apparent in maps of dust mass and dust temperature, which we constructed by fitting a modified blackbody to the observed spectral energy distribution in each sightline. Most of the dust that is seen is pre-existing interstellar dust in which SNRs leave imprints. The temperature maps clearly reveal SNRs heating surrounding dust, while the mass maps indicate the removal of $3.7_{-2.5}^{+7.5} M_{\odot}$ of dust per SNR. This agrees with the calculations by others that significant amounts of dust are sputtered by SNRs. Under the assumption that dust is sputtered and not merely pushed away, we estimate a dust destruction rate in the LMC of $0.037_{-0.025}^{+0.075} M_{\odot} \mathrm{yr}^{-1}$ due to SNRs, yielding an average lifetime for interstellar dust of $2_{-1.3}^{+4.0} \times 10^{7} \mathrm{yr}$. We conclude that sputtering of dust by SNRs may be an important ingredient in models of galactic evolution, that supernovae may destroy more dust than they produce, and that they therefore may not be net producers of long lived dust in galaxies.
\end{abstract}

Key words: dust, extinction - evolution - galaxies: ISM - ISM: clouds - ISM: supernova remnants Magellanic Clouds - submillimeter: galaxies - submillimeter: ISM

Supporting material: figure set

\section{INTRODUCTION}

Supernovæ ( $\mathrm{SNe}$ ) could be significant dust producers in galaxies, since around $0.1-1 M_{\odot}$ of dust can be produced in their ejecta as observations of some supernova remnants (SNRs; Barlow et al. 2010; Matsuura et al. 2011; Gomez et al. 2012a) and theoretical models suggest (Bianchi \& Schneider 2007; Nozawa et al. 2010). However, the amount of dust seen at high redshift is difficult to reconcile with dust forming in $\mathrm{SNe}$ alone (Silvia et al. 2010; Rowlands et al. 2014). There have been ample detections of dust created in SN ejecta shortly after the explosion (Elmhamdi et al. 2003; Fox et al. 2009; Matsuura et al. 2011), and in young SNRs such as Cas A (Nozawa et al. 2010), Crab (Gomez et al. 2012a; Temim et al. 2012; Temim
\& Dwek 2013), E 0102 (Stanimirović et al. 2005; Sandstrom et al. 2009), N 132D, and G 11.2-0.3 (Rho et al. 2009), but the inferred masses are generally well below theoretical predictions. While Gomez et al. (2012a) noticed the lack of dust production in Type Ia SNRs, and dust produced from Ic and Ib SNe has not been observed, there are indications for dust to be formed from IIn and IIP SNe (Gall 2010). However, for many SNe it is not certain whether the dust was pre-existing or formed in ejecta and some SNe were not detected (Szalai \& Vinkó 2013). SNe and SNRs also sputter dust in the surrounding interstellar medium (ISM) and pre-burst circumstellar medium (CSM). While it is well established that dust grows in evolved stars (e.g., AGB stars; see Boyer et al. 2012), it is not yet clear whether the net result of SNe and SNRs is a supply or removal of interstellar 
dust, and hence alternative solutions for dust growth are being considered, e.g., in the ISM (Zhukovska 2014).

The Large Magellanic Cloud (LMC) is a convenient place to study populations of SNRs because there is little foreground and internal contamination from interstellar clouds, the distances to all SNRs in the LMC are essentially identical and well known, and the LMC is close enough ( $\approx 50 \mathrm{kpc}$, Walker 2012) to resolve the far-infrared (FIR) and submillimeter emission of remnants with diameters $>9$ pc ( $>90 \%$ of known objects). Hence, SNRs in the LMC have been the subject of many detailed studies at all wavelengths. Here, we use the submillimeter data obtained as part of the HERITAGE (HERschel Inventory of The Agents of Galaxy Evolution) survey, (Meixner et al. 2013), covering 100-500 $\mu \mathrm{m}$, together with archival Spitzer Space Telescope data at 24 and $70 \mu \mathrm{m}$ from the Surveying the Agents of Galaxy Evolution (SAGE) LMC survey (Meixner et al. 2006), to quantify the influence of SNRs on the ISM of the LMC.

Many SNRs are detected at infrared (IR) wavelengths in the Galaxy and in the Magellanic Clouds (Reach et al. 2006; Seok et al. 2008, 2013; Williams et al. 2010). The radiation at $\lambda \gtrsim 24 \mu \mathrm{m}$ is mostly dust from swept-up ISM collisionally heated by the hot plasma generated by SNR shocks, while emission at shorter wavelengths originates from ionic/molecular lines, polycyclic aromatic hydrocarbons (PAH) emission, or synchrotron emission (Seok et al. 2013). The IR emission from SNRs may also include fine-structure line emission from hot plasma and/or shocks (van Loon et al. 2010) and the contributions from small grains that are stochastically heated and which may otherwise be rather cold.

However, the only detections of Magellanic SNRs at submillimeter wavelengths $(\lambda \gtrsim 100 \mu \mathrm{m})$ are SN 1987A, due to dust formed in the ejecta (Lakićević et al. 2011, 2012a, 2012b; Matsuura et al. 2011; Indebetouw et al. 2014) and LHA 120$\mathrm{N} 49$, explained by $10 M_{\odot}$ of dust in an interstellar cloud heated up by the forward shock (Otsuka et al. 2010). The submillimeter emission from SNRs could include a non-thermal (synchrotron) component from a strongly magnetized plasma, for instance, if a pulsar wind nebula (PWN) is present (Temim et al. 2012).

The most common ways for sputtering of grains in SNRs are thermal sputtering, when energetic particles knock atoms off the grain surface (Casoli \& Lequeux 1998), more often in fast shocks, $v>150 \mathrm{~km} \mathrm{~s}^{-1}$ and grain-grain collisions, dominant in slower shocks, $\leqslant 50-80 \mathrm{~km} \mathrm{~s}^{-1}$ (Jones et al. 1994), often called shattering. Sputtering is most effective on small grains (Andersen et al. 2011), resulting in a deficit of small grains in SNRs compared to the ISM. Shattering is destroying primarily big grains. Big grains (BG) become small (SG), which produces an increased SG-to-BG ratio (Andersen et al. 2011).

Sankrit et al. (2010) showed that $\sim 35 \%$ of dust is sputtered in the Cygnus Loop, a Galactic SNR, by modeling the flux ratio at 70 and $24 \mu \mathrm{m}$ in the post-shock region. Just behind the shock this ratio was 14 , compared to 22 further out from the remnant, which could be understood in terms of the destruction and heating of the dust by the shocks of this middle-aged SNR $(\sim 10,000 \mathrm{yr}-$ Blair et al. 2005). Arendt et al. (2010) showed that the interaction of the shock in young SNR Puppis A (3700 yr) with a molecular cloud has led to the destruction of $\approx 25 \%$ of the population of very small grains (PAH). Micelotta et al. (2010) explored the processing of PAHs in interstellar shocks by ion and electron collisions, finding that interstellar PAHs do not survive in shocks of velocities greater than $100 \mathrm{~km} \mathrm{~s}^{-1}$. Various other studies like Borkowski et al. (2006b) and Williams et al.
(2006, 2010), also found that significant amounts of dust were sputtered in the shocks of young SNRs.

For a homogeneous ISM and under the assumption that silicon and carbon grains are equally mixed, Dwek et al. (2007) obtained that the mass of the ISM, which is completely cleared of dust by one single SNR can be as high as $1200 M_{\odot}$. On the other hand, Bianchi \& Schneider (2007) predict $0.1 M_{\odot}$ of dust produced in SN to survive the passage of the reverse shock.

This paper is organized as follows. In Section 2, we introduce the sample of SNRs in the LMC, the data we analyze, and the methods we use. In Section 3, we present the results, in particular regarding the surface brightness, flux ratios, and dust mass and temperature maps. In Section 4, we discuss the implications, in terms of dust removal and the ISM properties within which the SNRs evolve. We summarize our conclusions in Section 5.

\section{DATA AND METHODS}

\subsection{Sample of Objects}

We examined 61 SNRs in the LMC, i.e., all objects that are relatively certain to be SNRs and that have accurate positions and dimensions selected using all existing survey catalogues: Williams et al. (1999); Blair et al. (2006); Seok et al. (2008); Payne et al. (2008); Badenes et al. (2010); Desai et al. (2010); Maggi et al. (2014), and an unpublished catalog of Filipović et al. The complete sample of targets, their positions and sizes are given in Table 1, with more details for SNRs for which ages and types have been determined in Table 2 .

The explosion type is often not known or is uncertain and the assumed core-collapse (CC) SNRs in our sample may still harbor some Ia, while the group of assumed Ias are the SNRs that are considered to be Ias in the literature.

\subsection{FIR and Submillimeter Data}

We use FIR and submillimeter data from the Herschel Space Observatory open time key program, HERITAGE (Meixner et al. 2013), of the LMC, comprising images obtained with SPIRE (Spectral and Photometric Imaging Receiver) at 250, 350 , and $500 \mu \mathrm{m}$ and with PACS (Photodetector Array Camera and Spectrometer) at 100 and $160 \mu \mathrm{m}$. We complement this with IR images from the SAGE project, of the LMC (Meixner et al. 2006), obtained with the MIPS instrument on board the Spitzer Space Telescope at 24 and $70 \mu \mathrm{m}$.

For making mass and temperature maps, we use Herschel images at 100, 160, 250, 350, and $500 \mu \mathrm{m}$ from Gordon et al. (2014). These images have the same resolution (36."3), which is the limiting resolution of the $500 \mu \mathrm{m}$ band, are projected to the same pixel size $\left(14^{\prime \prime}\right)$ and are subtracted for the residual foreground Milky Way cirrus emission and the confusion noise of unresolved background galaxies. In Figure 1, we show an example of these data for one object-SNR N 49. For the ratio maps and the rest of this paper we also use similar maps of MIPS data at 24 and $70 \mu \mathrm{m}$.

The errors in the MIPS data are calculated by adding in quadrature the flux calibration uncertainties of $2 \%$ and $5 \%$ (for 24 and $70 \mu \mathrm{m}$ data) and background noise measured away from the galaxy. Likewise, errors in the PACS and SPIRE images were found by combining in quadrature, respectively $10 \%$ and $8 \%$ uncertainties of the absolute flux calibration (Meixner et al. 2013) with the background noise described by Gordon et al. (2014). In their work it is conservatively assumed that the uncertainties between bands were not correlated and the impact of this assumption on the resulting dust masses is discussed. 
Table 1

Complete Sample of SNRs in the LMC

\begin{tabular}{|c|c|c|c|}
\hline Name & $\begin{array}{l}\text { R.A. }^{\mathrm{a}} \\
\text { (h m s) }\end{array}$ & $\begin{array}{l}\text { Decl. }^{\mathrm{a}} \\
\left({ }^{\circ} \prime \prime \prime\right)\end{array}$ & $\begin{array}{l}D^{\mathrm{a}} \\
\left({ }^{\prime \prime}\right)\end{array}$ \\
\hline J 0448.4-6660 & 044822 & -665952 & 220 \\
\hline J $0449.3-6920$ & 044920 & -692020 & 133 \\
\hline В $0449-693^{\mathrm{b}}$ & 044940 & -692149 & 120 \\
\hline B $0450-6927$ & 045015 & -692212 & 210 \\
\hline В $0450-709$ & 045027 & -705015 & 357 \\
\hline LHA 120-N 4 (N 4) & 045314 & -665513 & 252 \\
\hline $0453-68.5$ & 045338 & -682927 & 120 \\
\hline B $0454-7000$ & 045352 & -700013 & 420 \\
\hline LHA 120-N9 (N9) & 045433 & -671313 & 177 \\
\hline LHA 120-N 11L (N 11L) & 045449 & -662532 & 87 \\
\hline LHA 120-N 86 (N 86) & 045537 & -683847 & 348 \\
\hline LHA 120-N 186D (N 186D) & 045955 & -700752 & 150 \\
\hline DEM L71 & 050542 & -675239 & 72 \\
\hline LHA 120-N 23 (N 23) & 050555 & -680147 & 111 \\
\hline J $0506.1-6541$ & 050605 & -654108 & 408 \\
\hline В $0507-7029$ & 050650 & -702553 & 330 \\
\hline RXJ $0507-68^{\mathrm{b}}$ & 050730 & -684700 & 450 \\
\hline $\mathrm{J} 0508-6830^{\mathrm{d}}$ & 050849 & -683041 & 123 \\
\hline LHA $120-\mathrm{N} 103 \mathrm{~B}$ (N 103B) & 050859 & -684335 & 28 \\
\hline $0509-67.5$ & 050931 & -673117 & 29 \\
\hline J0511-6759d & 051111 & -675907 & 108 \\
\hline DEM L109 & 051314 & -691220 & 215 \\
\hline $\mathrm{J} 0514-6840^{\mathrm{d}}$ & 051415 & -684014 & 218 \\
\hline $\mathrm{J} 0517-6759^{\mathrm{d}}$ & 051710 & -675903 & 270 \\
\hline LHA 120-N 120 (N 120) & 051841 & -693912 & 134 \\
\hline $0519-69.0$ & 051935 & -690209 & 31 \\
\hline $0520-69.4$ & 051944 & -692608 & 174 \\
\hline J $0521.6-6543$ & 052139 & -654307 & $162^{e}$ \\
\hline LHA 120-N 44 (N 44) & 052307 & -675312 & 228 \\
\hline LHA 120-N 132D (N 132D) & 052504 & -693824 & 114 \\
\hline LHA 120-N 49B (N 49B) & 052525 & -655919 & 168 \\
\hline LHA 120-N 49 (N 49) & 052600 & -660457 & 84 \\
\hline В $0528-692$ & 052739 & -691204 & 147 \\
\hline DEM L 204 & 052754 & -654938 & 303 \\
\hline HP99498 & 052820 & -671340 & 97 \\
\hline B $0528-7038$ & 052803 & -703740 & 60 \\
\hline DEM L203 & 052905 & -683230 & 667 \\
\hline DEM L214 & 052951 & -670105 & 100 \\
\hline DEM L $214^{\mathrm{c}}$ & 052952 & -665331 & 120 \\
\hline DEM L218 & 053040 & -700730 & 213 \\
\hline LHA 120-N 206 (N 206) & 053156 & -710019 & 192 \\
\hline $0532-67.5$ & 053230 & -673133 & 252 \\
\hline В $0534-69.9$ & 053402 & -695503 & 114 \\
\hline DEM L238 & 053418 & -703326 & 180 \\
\hline SN 1987A & 053528 & -691611 & 2 \\
\hline LHA 120-N 63A (N 63A) & 053544 & -660214 & 66 \\
\hline Honeycomb & 053546 & -691802 & 102 \\
\hline DEM L241 & 053603 & -673435 & 135 \\
\hline DEM L249 & 053607 & -703837 & 180 \\
\hline B $0536-6914$ & 053609 & -691153 & 480 \\
\hline DEM L256 & 053727 & -662750 & 204 \\
\hline B $0538-6922$ & 053737 & -692023 & 169 \\
\hline $0538-693^{\mathrm{b}}$ & 053814 & -692136 & 169 \\
\hline LHA 120-N 157B (N 157B) & 053746 & -691028 & 102 \\
\hline LHA 120-N 159 (N 159) & 053959 & -694402 & 78 \\
\hline LHA 120-N 158A (N 158A) & 054011 & -691955 & 60 \\
\hline DEM L299 & 054308 & -685818 & 318 \\
\hline DEM L316B & 054659 & -694250 & 84 \\
\hline DEM L316A & 054722 & -694126 & 56 \\
\hline $0548-70.4$ & 054749 & -702454 & 102 \\
\hline J $0550.5-6823$ & 055030 & -682240 & 312 \\
\hline
\end{tabular}

Notes.

${ }^{a}$ From Badenes et al. (2010), if not written differently.

b This SNR is from the Blair et al. (2006) catalogue.

c This SNR is from Filipović's unpublished catalogue.

${ }^{\mathrm{d}}$ From Maggi et al. (2014).

${ }^{\mathrm{e}}$ From Desai et al. (2010).

\section{RESULTS}

\subsection{Evolution of the FIR, Submillimeter and Radio Surface Brightness and Diameter with SNR Age}

We first examine the FIR and radio surface brightness with regard to SNR diameters, to check if there is any evolution in the time. In this analysis, we do not apply color corrections. The radio surface brightness is obtained from

$$
\Sigma_{\nu}\left[\mathrm{Wm}^{-2} \mathrm{~Hz}^{-1} \mathrm{sr}^{-1}\right]=1.505 \times 10^{-19} \frac{F_{\nu}[\mathrm{Jy}]}{\Theta\left[^{\prime}\right]^{2}},
$$

where $F_{v}$ is the flux density and $\Theta$ the angular diameter of a source (Vukotić et al. 2009). Surface brightness of SNRs is decreasing with diameter at radio wavelengths because the objects spread, cool down, and mix with ISM.

By performing linear fitting on radio data at $1.4 \mathrm{GHz}$, we have found the $\Sigma-D$ relation to be

$$
\Sigma_{1.4 \mathrm{GHz}}=\left(1.2_{-0.9}^{+3.7}\right) \times 10^{-17} \times D^{-2.1 \pm 0.4},
$$

where the diameter $D$ is in parsecs, with a correlation coefficient of -0.7 , using 26 SNRs (see Table 2) for which we have radio fluxes from Badenes et al. (2010) catalogue and an estimation of the age in the literature. These data are presented in the top left panel of Figure 2. This agrees well with that found in the literature (Arbutina et al. 2004; Urošević 2003) and suggests a relatively constant radio luminosity. Similarly, in the top right panel is the dependence of $\Sigma_{1.4 \mathrm{GHz}}$ of the age of the remnant for which we have found the relation

$$
\left.\Sigma_{1.4 \mathrm{GHz}}=\left(0.27_{-0.25}^{+2.29}\right) \times 10^{-16} \times \mathrm{age}_{\mathrm{yr}}\right]^{-0.86 \pm 0.25},
$$

with a correlation coefficient of -0.58 . This is consistent with a time evolution of the diameter according to $D \propto \operatorname{age}^{0.41}$, or almost exactly the age $\mathrm{e}^{2 / 5}$ as predicted for the Sedov phase (see, e.g., Badenes et al. 2010).

Similarly, we compared the surface brightness at FIR wavelengths $\left(\Sigma_{24}, \Sigma_{160}, \Sigma_{350}\right.$, and $\left.\Sigma_{500}\right)$ and the diameters, but no correlation was found (see Table 3). It is most likely that the FIR emission is dominated by the ISM rather than the SNR.

However, for the relation between FIR and radio surface brightness there is some tentative trend (see Figure 2 bottom left for the $\Sigma_{160}$ ). This is a consequence of the dependence of both the $\Sigma_{\text {radio }}$ and $\Sigma_{\text {FIR }}$ on the density of the ISM. We find similar correlations between the $\Sigma_{1.4 \mathrm{GHz}}$ and that at other FIR and submillimeter wavelengths (Table 3 ). The correlation coefficients are similar for all of these frequencies $(0.55-0.7)$. Comparison between $\Sigma_{24}$ and $\Sigma_{1.4 \mathrm{GHz}}$ is given in Figure 2 in the bottom right panel. Somewhat surprising is the much weaker correlation than the one obtained by Seok et al. (2008) who found the correlation coefficient between $\Sigma_{24}$ and $\Sigma_{\text {radio }}$ to be 0.98 , using only eight clearly detected SNRs.

The $24 \mu \mathrm{m}$ flux may have a contribution from line emission. Among the SNRs believed to be more affected by this are N 49 and $\mathrm{N} 63 \mathrm{~A}$, where the line contribution is estimated to be an exceptional $\sim 80 \%$ (Williams et al. 2006) and modest $\sim 10 \%$ (Caulet et al. 2012), respectively. We corrected $\Sigma_{24}$ for these two SNRs accordingly. In general, however, line emission is thought to make a negligible contribution to the $24 \mu \mathrm{m}$ flux (Williams et al. 2010) and we found estimates in the literature only for these two SNRs.

Since we have not found any fading of $\Sigma_{\text {FIR }}$ with the diameter, we will try to find it in the following experiment. In Figure 3(a) 
Table 2

The LMC SNRs with Known Age and Type from the Literature

\begin{tabular}{|c|c|c|c|c|c|c|c|c|}
\hline Name & $\begin{array}{l}\text { R.A. }^{a} \\
\text { (h m s) }\end{array}$ & $\begin{array}{l}\text { Decl. }^{\mathrm{a}} \\
{\left[{ }^{0},{ }^{\prime \prime}\right]}\end{array}$ & $\begin{array}{c}D^{\mathrm{a}} \\
(\mathrm{pc})\end{array}$ & $\begin{array}{l}\text { Age } \\
(\mathrm{yr})\end{array}$ & Ref. Age & $\begin{array}{c}F_{1.4 \mathrm{GHz}}{ }^{\mathrm{a}} \\
(\mathrm{Jy})\end{array}$ & Type & Ref. Type \\
\hline $0450-70.9$ & 045027 & -705015 & 86 & $\leqslant 45000$ & 26 & 0.56 & $\mathrm{CC} ?$ & 27 \\
\hline В $0453-68.5$ & 045338 & -682927 & 29 & 13000 & 1 & 0.11 & $\mathrm{CC}$ & 1 \\
\hline N9 & 045433 & -671313 & 43 & 30000 & 24 & 0.06 & Ia? & 24 \\
\hline N 11L & 045449 & -662532 & 21 & 11000 & 2 & 0.11 & $\mathrm{CC}$ & 9 \\
\hline N 86 & 045537 & -683847 & 84 & 86000 & 2 & 0.26 & $\mathrm{CC} ?$ & 2 \\
\hline N 23 & 050555 & -680147 & 27 & 6300 & 3,4 & 0.35 & $\mathrm{CC}$ & 5 \\
\hline N 103B & 050859 & -684335 & 7 & 1000 & 6 & 0.51 & Ia & 6 \\
\hline В $0509-67.5$ & 050931 & -673117 & 8 & 400 & 7 & 0.08 & Ia & 7 \\
\hline N 120 & 051841 & -693912 & 32 & 7300 & 8 & 0.35 & $\mathrm{CC}$ & 9 \\
\hline В $0519-69.0$ & 051935 & -690209 & 8 & 600 & 7 & 0.1 & $\mathrm{Ia}$ & 7 \\
\hline N 44 & 052307 & -675312 & 55 & 18000 & 10 & 0.14 & $\mathrm{CC} ?$ & 28 \\
\hline N 132D & 052504 & -693824 & 28 & 2750 & 3 & 3.71 & $\mathrm{CC}$ & 11 \\
\hline N 49 & 052600 & -660457 & 20 & 6600 & 13 & 1.19 & $\mathrm{CC}$ & 14 \\
\hline N 206 & 053156 & -710019 & 46 & 25000 & 15 & 0.33 & $\mathrm{CC}$ & 15 \\
\hline $0534-69.9$ & 053402 & -695503 & 27 & 10000 & 16 & 0.08 & Ia & 16 \\
\hline DEM L238 & 053418 & -703326 & 43 & 13500 & 17 & 0.06 & Ia & 17 \\
\hline SN 1987A & 053528 & -691611 & 0.5 & 27 & 18 & 0.05 & $\mathrm{CC}$ & 18 \\
\hline N63A & 053544 & -660214 & 16 & 3500 & 19 & 1.43 & $\mathrm{CC}$ & 19 \\
\hline DEM L249 & 053607 & -703837 & 43 & 12500 & 17 & 0.05 & Ia & 17 \\
\hline N 157B & 053746 & -691028 & 25 & 5000 & 20 & 2.64 & $\mathrm{CC}$ & 20 \\
\hline N 159 & 053959 & -694402 & 19 & 18000 & 21 & 1.9 & $\mathrm{CC}$ & 21 \\
\hline B $0540-69.3$ & 054011 & -691955 & 15 & 800 & 22 & 0.88 & $\mathrm{CC}$ & 5 \\
\hline DEM L316A & 054722 & -694126 & 14 & 33000 & 23 & 0.33 & Ia & 23 \\
\hline В $0548-70.4$ & 054749 & -702454 & 25 & 7100 & 3 & 0.05 & $\mathrm{CC}$ & 3 \\
\hline DEM L241 & 053603 & -673435 & 33 & 12000 & 25 & 0.29 & $\mathrm{CC}$ & 25 \\
\hline
\end{tabular}

Note.

${ }^{a}$ From Badenes et al. (2010).

References. (1) Haberl et al. (2012); (2) Williams et al. (1999); (3) Williams et al. (2010); (4) Someya et al. (2010); (5) Hayato et al. (2006); (6) Lewis et al. (2003); (7) Rest et al. (2005); (8) Rosado et al. (1993); (9) Chu \& Kennicutt (1988); (10) Chu et al. (1993); (11) Vogt \& Dopita (2011); (12) Park et al. (2003b); (13) Park et al. (2003a); (14) Otsuka et al. (2010); (15) Williams et al. (2005); (16) Hendrick et al. (2003); (17) Borkowski et al. (2006a); (18) Zanardo et al. (2010); (19) Hughes et al. (1998); (20) Wang et al. (2001); (21) Seward et al. (2010); (22) Badenes et al. (2009); (23) Williams et al. (2005); (24) Seward et al. (2006); (25) Seward et al. (2012); (26) Williams et al. (2004); (27) The SNR appears centrally filled in X-rays and radio, but no point source is detected in either radio or X-ray observations (Williams et al. 2004). (28) The remnant is near H II complexes and OB associations (Chu et al. 1993).

Table 3

Relations between Surface Brightness $(\Sigma)$ and SNR Diameters

\begin{tabular}{lccccccc}
\hline \hline $\log \Sigma$ & \multicolumn{3}{c}{$\log D(\mathrm{pc})$} & & \multicolumn{3}{c}{$\log \Sigma_{1.4 \mathrm{GHz}}$} \\
\cline { 2 - 4 } \cline { 6 - 7 } & c. corr. & $A$ & $B$ & & c. corr. & $A$ & $B$ \\
\hline $\log \Sigma_{24}$ & -0.3 & $-18.53 \pm 0.78$ & $-0.85 \pm 0.55$ & & 0.7 & $-6.61 \pm 2.71$ & $0.66 \pm 0.14$ \\
$\log \Sigma_{160}$ & -0.14 & $-17.88 \pm 0.58$ & $-0.29 \pm 0.41$ & & 0.59 & $-10.49 \pm 2.19$ & $0.39 \pm 0.11$ \\
$\log \Sigma_{350}$ & -0.09 & $-18.67 \pm 0.51$ & $-0.16 \pm 0.36$ & & 0.55 & $-12.46 \pm 1.98$ & $0.3 \pm 0.1$ \\
$\log \Sigma_{500}$ & -0.08 & $-23.08 \pm 0.49$ & $-0.14 \pm 0.34$ & & 0.56 & $-17.09 \pm 1.89$ & $0.31 \pm 0.09$ \\
$\log \Sigma_{1.4 \mathrm{GHz}}$ & -0.7 & $-16.94 \pm 0.63$ & $-2.1 \pm 0.4$ & & & & \\
\hline
\end{tabular}

Note. For these linear relations we tabulate the correlation coefficient, constant $A$ and slope $B$, where $\log y=A+B \log x$.

we first plot the surface brightness, normalized to $\Sigma_{250}$ to remove variations between SNRs for other reasons than evolution, versus diameter. We then smooth the data over up to \pm 7 consecutive measurements, revealing clear trends of fading with time (Figure 3(b)). It appears as though there is no further evolution for SNRs with $D>70 \mathrm{pc}$.

While the fading at $24 \mu \mathrm{m}$ is the most prominent evolution across the IR-submillimeter range (a factor of three change over our sample, compared to $\Sigma_{250}$ ), less expected is the fading at submillimeter wavelengths $(350$ and $500 \mu \mathrm{m})$, by a factor of two compared to $\Sigma_{250}$-i.e., more pronounced than at 100 and $160 \mu \mathrm{m}$. Perhaps free-free and/or synchrotron emission contributes at the longest wavelengths, and diminishes during the evolution of the SNR. As the SED peaks shortward of $350 \mu \mathrm{m}$, estimates of the dust mass and temperature will not be greatly affected by such contributions at longer wavelengths.

Figure 3(c) shows the same as for the previous panel, but in the $20 \mathrm{pc}$ thick annuli just outside of SNRs. It suggests that the environment of SNRs also fades and that, on average, surface brightnesses within SNRs are not much different from the ones 

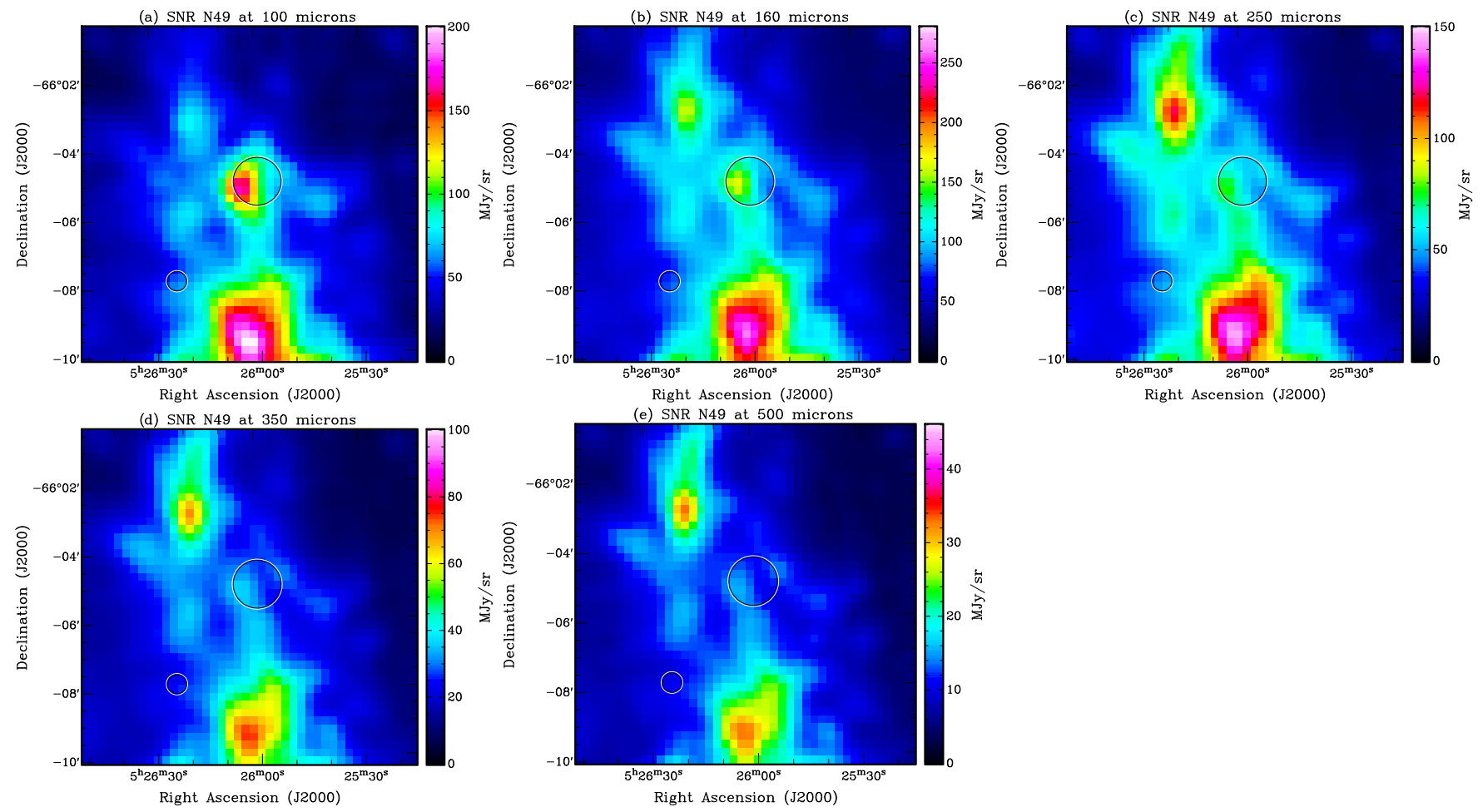

Figure 1. Images of SNR N 49: (a) at 100, (b) at 160, (c) at 250, (d) at 350, and (e) at $500 \mu \mathrm{m}$, shown on the resolution of $366^{\prime \prime} 3$. Beam size is presented by the circle in the lower left corner and the remnant dimension and position by the circle in the middle.
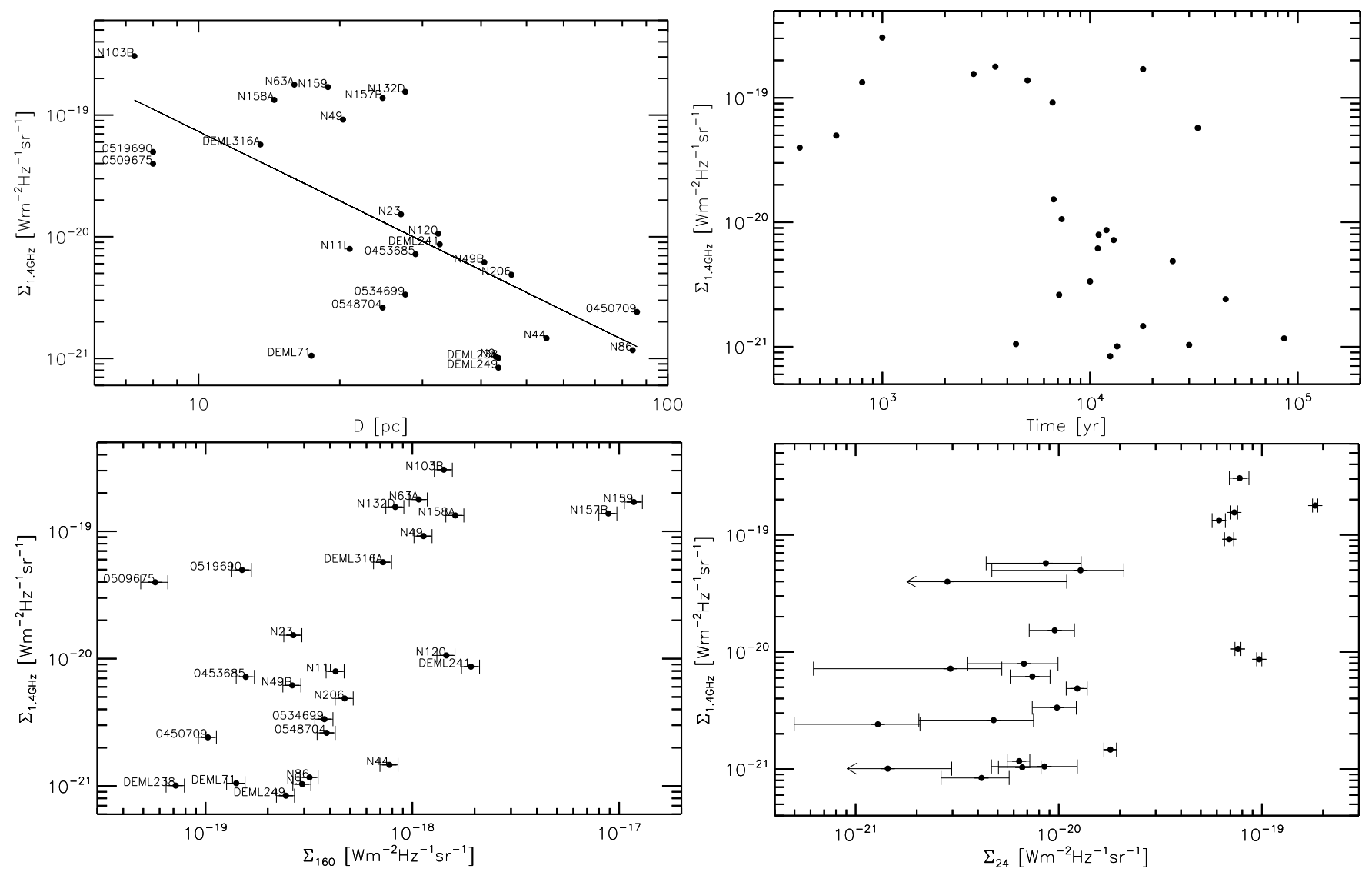

Figure 2. Top left panel: relation between SNR $\Sigma_{1.4 \mathrm{GHz}}$ and diameter of SNR, $\Sigma-D$ relation. Top right panel: relation between SNR $\Sigma_{1.4 \mathrm{GHz}}$ and the age of SNR. Bottom left: $\Sigma_{1.4 \mathrm{GHz}}$ is weakly correlated with $\Sigma_{160}$. Bottom right: $\Sigma_{1.4 \mathrm{GHz}}$ has some correlation with $\Sigma_{24}$. 

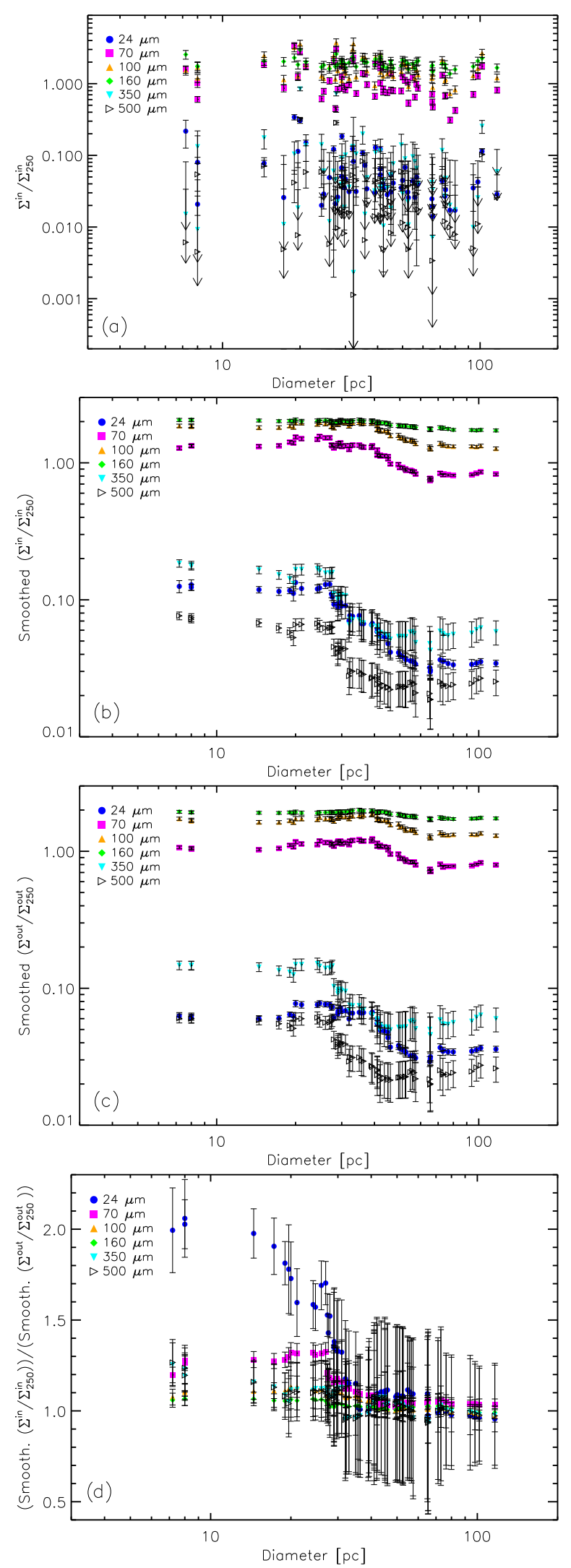

Figure 3. (a) Evolution of $\Sigma$ (normalized to $\Sigma_{250}$ ) within SNRs at $24-500 \mu \mathrm{m}$ depending on the diameter; (b) the same as panel (a), but smoothed; (c) the same as previous, but in annuli around SNRs. (d) Ratio of normalized and smoothed $\Sigma$ inside and outside of the SNR. from annuli. This might be a sign of the cooling of the dust in the ISM.

Finally, in Figure 3(d), we derive the ratio of inner and outer normalized and smoothed $\Sigma$, finding that most of the wavelengths of SNRs show some decrease in $\Sigma$ compared to the surroundings.

\subsection{Maps of Flux Ratio}

Following Sankrit et al. (2010) and Williams et al. (2010) who used IR flux ratios to infer the sputtering and heating of dust in SNR shocks, we constructed maps of the flux ratios at 70 and $24 \mu \mathrm{m}$ (hereafter $\left.R_{70 / 24}\right)$ and at 350 and $70 \mu \mathrm{m}\left(R_{350 / 70}\right)$. Maps using other combinations of 24-500 $\mu \mathrm{m}$ fluxes where dividend flux is at a longer wavelength than that of the divisor do not show any different behavior-ratios within SNRs are lower than in the surrounding medium. We show the images of these two ratios for N 49 and N 49B in Figure 4. In making these maps, we do not apply color correction.

The differences between $R_{70 / 24}$ (and $R_{350 / 70}$ ) within and outside of the SNRs result from differences in the warm dust contribution (and in some cases line emission around $24 \mu \mathrm{m}$ ), but also from the sputtering of the dust (Sankrit et al. 2010; Williams et al. 2010). This warm dust is collisionally heated (Williams et al. 2010), dominated by small grains, and thus sensitive to the effect of thermal or non-thermal sputtering and shattering.

We calculate the ratios between the average flux ratio outside (in $20 \mathrm{pc}$ thick annuli) and within the SNR, which we shall call $R_{70 / 24}^{\text {out } / \text { in }}$ and $R_{350 / 70}^{\text {out } / \text { in }}$, respectively, and plot these against the ages of objects (if they are known) and diameters (Figure 5). There is a clear trend of the $R_{70 / 24}$ ratio within the SNR to increase as the SNR evolves, until it reaches that of the surroundings. This suggests the dust within/around the SNR cools in $\sim 10^{4}$ yr. The older SNRs show little difference from the surrounding ISM, yet the difference still lingers as the ratio-ratio stays above unity for most SNRs, also in the $R_{350 / 70}$ ratio. The same observations are reflected in the dependence on diameter (Figure 5, bottom panels). $R_{70 / 24}^{\text {out/in }}$ is more sensitive to the SNR temperature, while $R_{350 / 70}^{\text {out } / \text { in }}$ is also sensitive to the dust mass that SNR interacts with.

\subsection{Creating Maps of Dust Mass and Temperature}

For producing mass and temperature maps, we use only the pixels with flux higher than $3 \sigma$ where the uncertainties are different for each wavelength and pixel. If the flux of a pixel is below that limit, then it is added to the fluxes of all other faint pixels in that image and the value of the average faint pixel is found, which is then fit using only the calibration uncertainties (as the background uncertainties become negligible).

We assume a modified black body in the shape

$$
F_{v} \propto v^{\beta} B_{v}
$$

using the emissivity index $\beta=1.5$ (see Planck Collaboration et al. 2014), where $B_{v}$ is Planck function and $v$ frequency. We use the emissivity $\kappa=0.1 \mathrm{~m}^{2} \mathrm{~kg}^{-1}$ at $\lambda=1 \mathrm{~mm}$ wavelength (Mennella et al. 1998). The two free parameters are the temperature and mass, initially these were set to $T=20 \mathrm{~K}$ (see Planck Collaboration et al. 2014, who derived $T \approx 18.7 \mathrm{~K}$ ) and $M=1 M_{\odot}$. We further constrained the temperature to be $\geqslant 3 \mathrm{~K}$ (cosmic microwave background) and $\leqslant 25,000 \mathrm{~K}$-all grains will have sublimated by $T \sim 1500 \mathrm{~K}$ (the solution for $T$ is always well below this physical limit); and the mass 

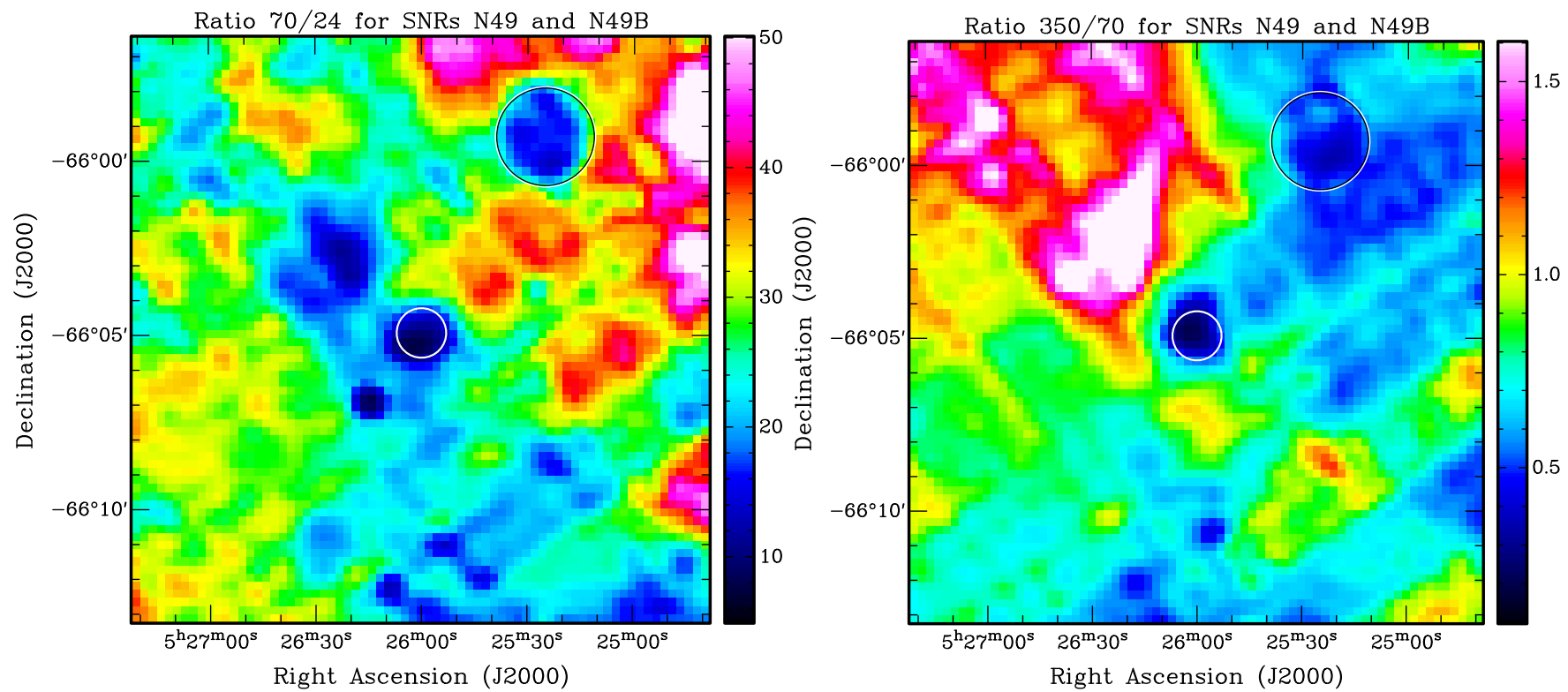

Figure 4. Maps of flux ratios for $\mathrm{N} 49$ (central circle is the surface of the remnant) and N 49B (circle on top right). Left: $R_{70 / 24}$; right: $R_{350 / 70}$. Color correction was not applied.
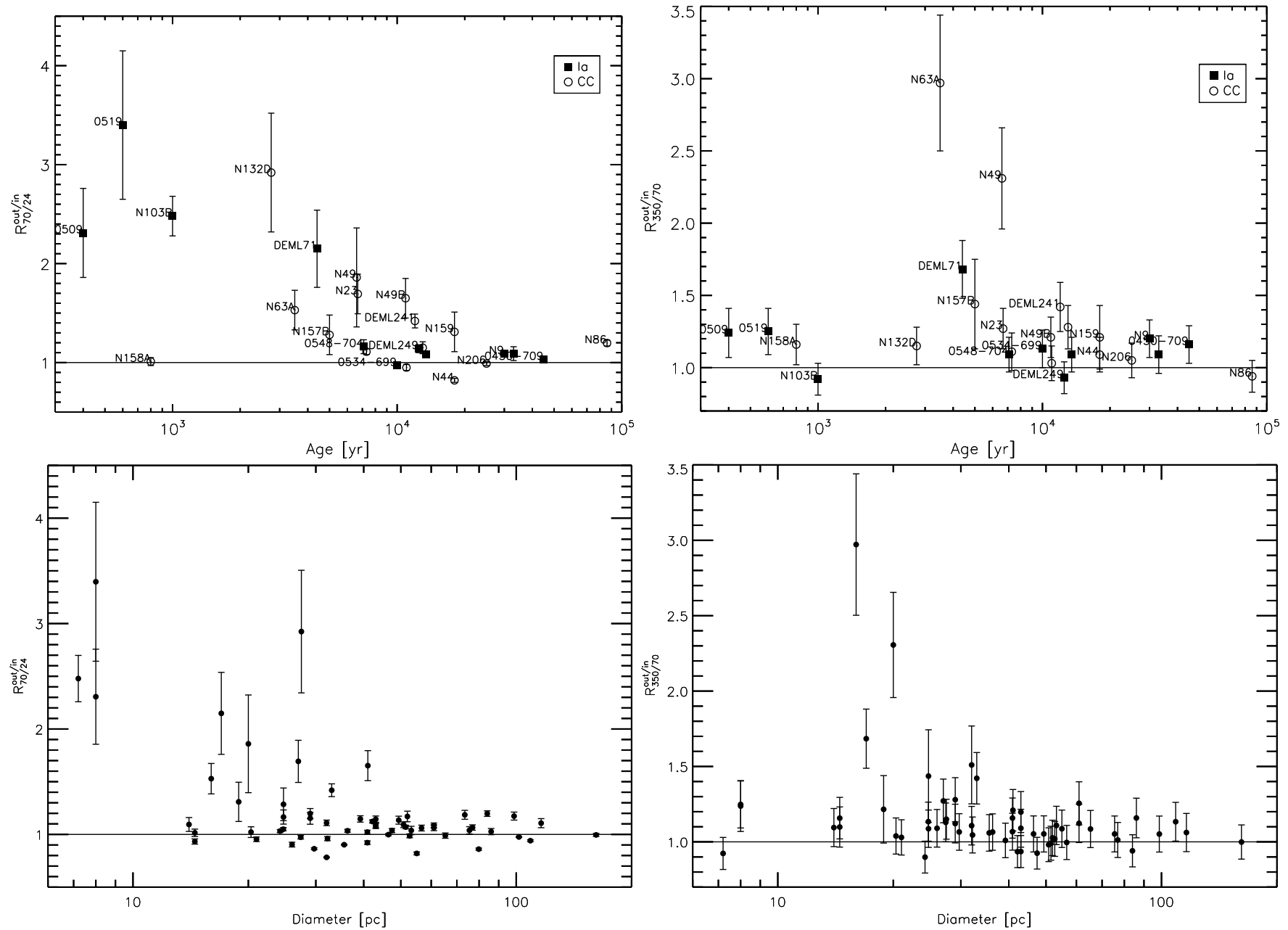

Figure 5. Top: evolution of the average ratio of IR fluxes (70 and $24 \mu \mathrm{m})$ outside divided by the one within the SNR; left and right show $R_{70 / 24}^{\text {out } / \text { in }}$ and $R_{350 / 70}^{\text {out }}$ in time. Bottom: likewise for the dependence on diameter, for the entire sample of SNRs. 

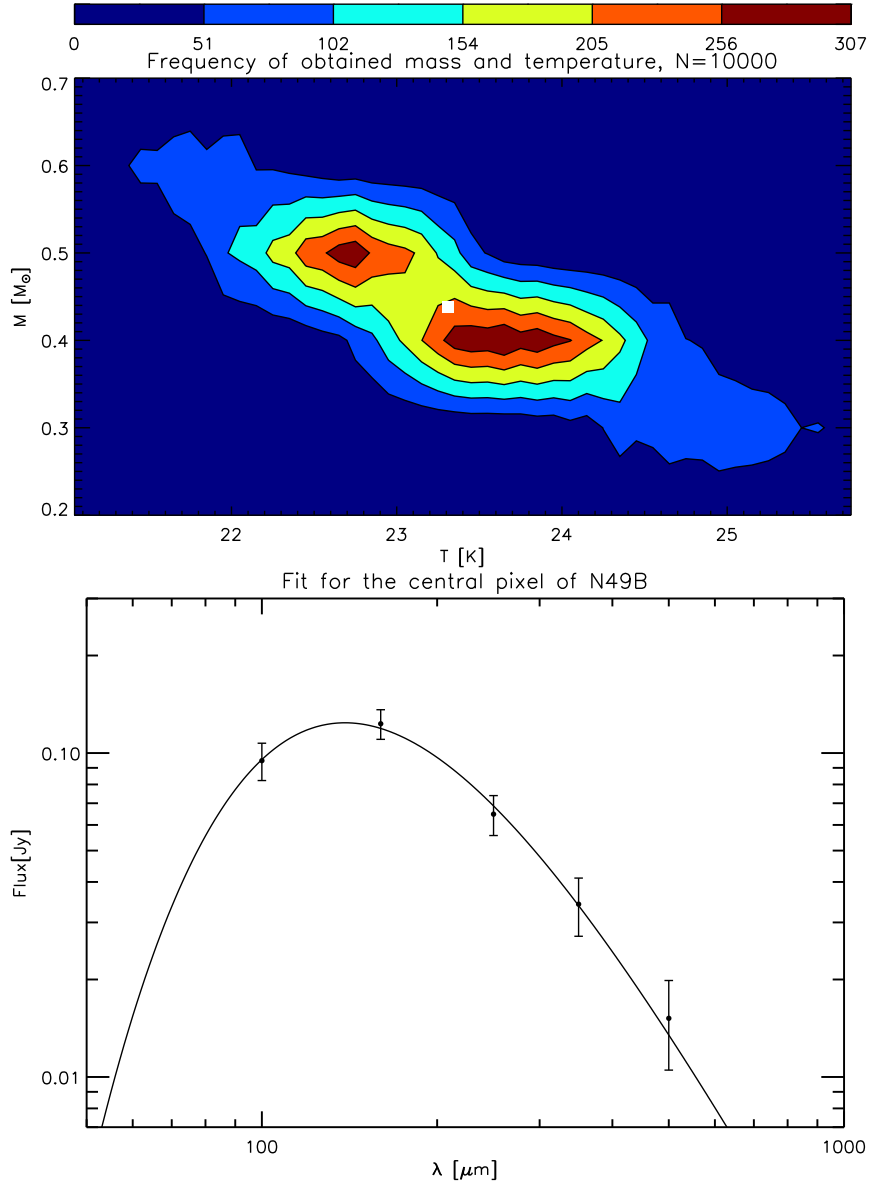

Figure 6. Top: the obtained values of mass and temperature for the central pixel in the image of $\mathrm{N} 49 \mathrm{~B}$, based on fits to 10,000 simulated data sets. The white square is the actual result of the fit for that pixel. Bottom: fitting of the SED of that particular pixel.

to be $\geqslant 10^{-5} M_{\odot}$ and $\leqslant 180 M_{\odot}$. Each pixel was modeled separately, using a $\chi^{2}$ minimization procedure within IDL (MPFIT, Markwardt 2009).

First, we performed the fitting without color correction, then we computed the color corrections to that model, as an iterative search for a best dust model. Color correction is computed using the IDL wrapper distributed with the DusTEM code and described in Compiègne et al. (2011). Finally, we applied the fit to data that were divided by that color correction. As a result, we obtained temperature and mass maps for every SNR and surroundings.

The errors of these maps can be estimated using Monte Carlo simulations or using MPFIT. Monte Carlo simulation is done by adding random Gaussian noise with standard deviation equal to the error on the fluxes, and fitting the resulting SED. In the contour plot in Figure 6, top, is the distribution of values of mass and temperature using 10,000 simulated data sets. The white square marks the actual fit for the given pixel. In Figure 6, bottom, is the actual fit for the given pixel.

In Figure 7, we show the actual histograms of obtained values of mass and temperature for the same 10,000 data sets. From the width of the Gaussians, we find the errors of the mass and the temperature for that pixel of $6.9 \%$ and $1.8 \%$ respectively. Average $\chi^{2}$ was 3.75 . With five data points and two fit parameters, the number of degrees of freedom is three. These errors are different from pixel to pixel and from remnant to

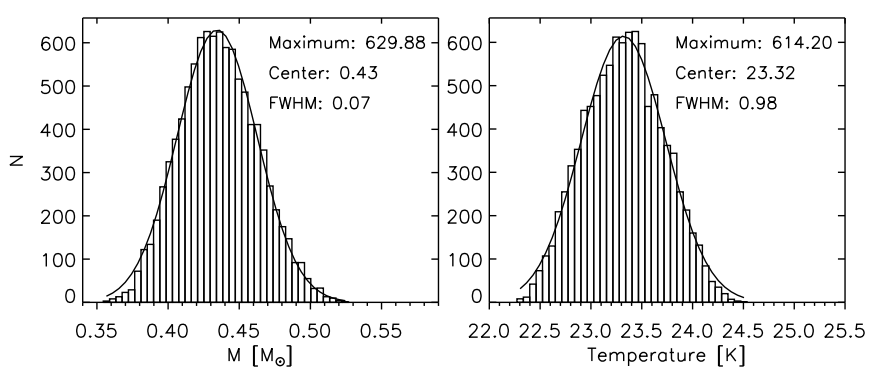

Figure 7. Histograms of the (Left) mass and (Right) temperature obtained for the 10,000 simulated data sets around the central pixel of SNR N49B. From FWHM we can find the values of $\sigma$.
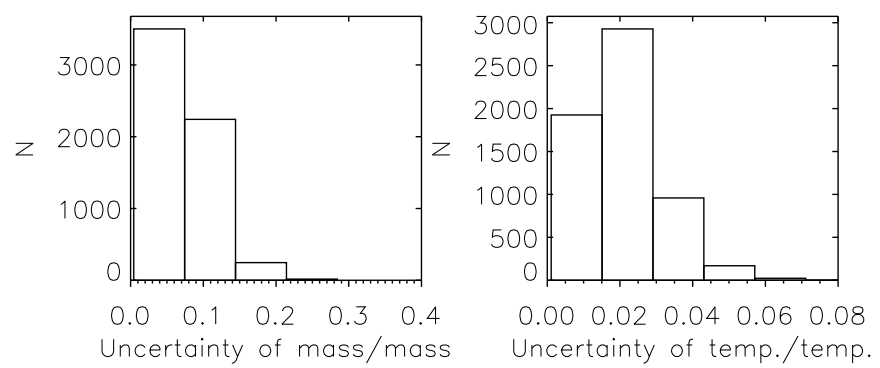

Figure 8. Histograms presenting the ratio of uncertainties of parameters and the parameters themselves for pixels in the N 49B image, for (left) mass and (right) temperature.

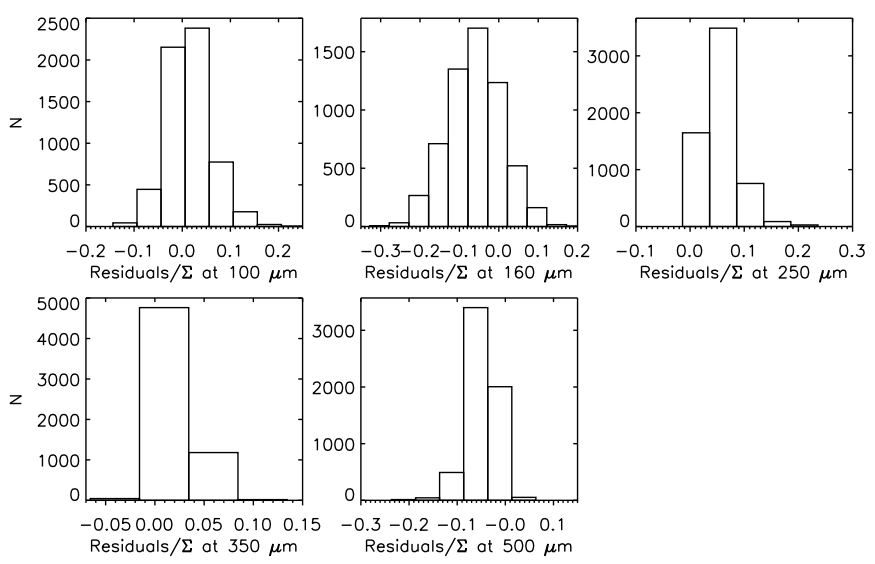

Figure 9. Histograms of the residual $/ \Sigma$ (for all pixels) on all wavelengths for SNR N 49B and its surroundings. These residuals are of the order of the surface brightness uncertainties $(\sim 10 \%)$ and therefore our adopted model describes the data well.

remnant. For low density SNRs, like 0506-675 and DEM L71 we cannot use this method for finding errors since the flux is too low (hardly above $3 \sigma$ ).

Because Monte Carlo simulations for every pixel and remnant would be time consuming, we find the uncertainties of mass and temperature from the MPFIT procedure itself. In Figure 8, we give the distributions of the ratios of the uncertainties and corresponding parameters, for mass and temperature, respectively, for SNR N 49B and surroundings, using 9120 pixels, or $320 \times 320 \mathrm{pc}^{2}$. The maps of N 49B and surroundings are representative for the LMC because there are enough faint and bright pixels to estimate the error distribution. These uncertainties are $<40 \%$ for the mass and $<13 \%$ for the temperature. The pixel averaged $\chi^{2}$ was 1.57 .

In Figure 9, we show the histograms of the residuals (model minus data) divided by corresponding surface brightnesses at all wavelengths for SNR N 49B and the surroundings. The residuals 

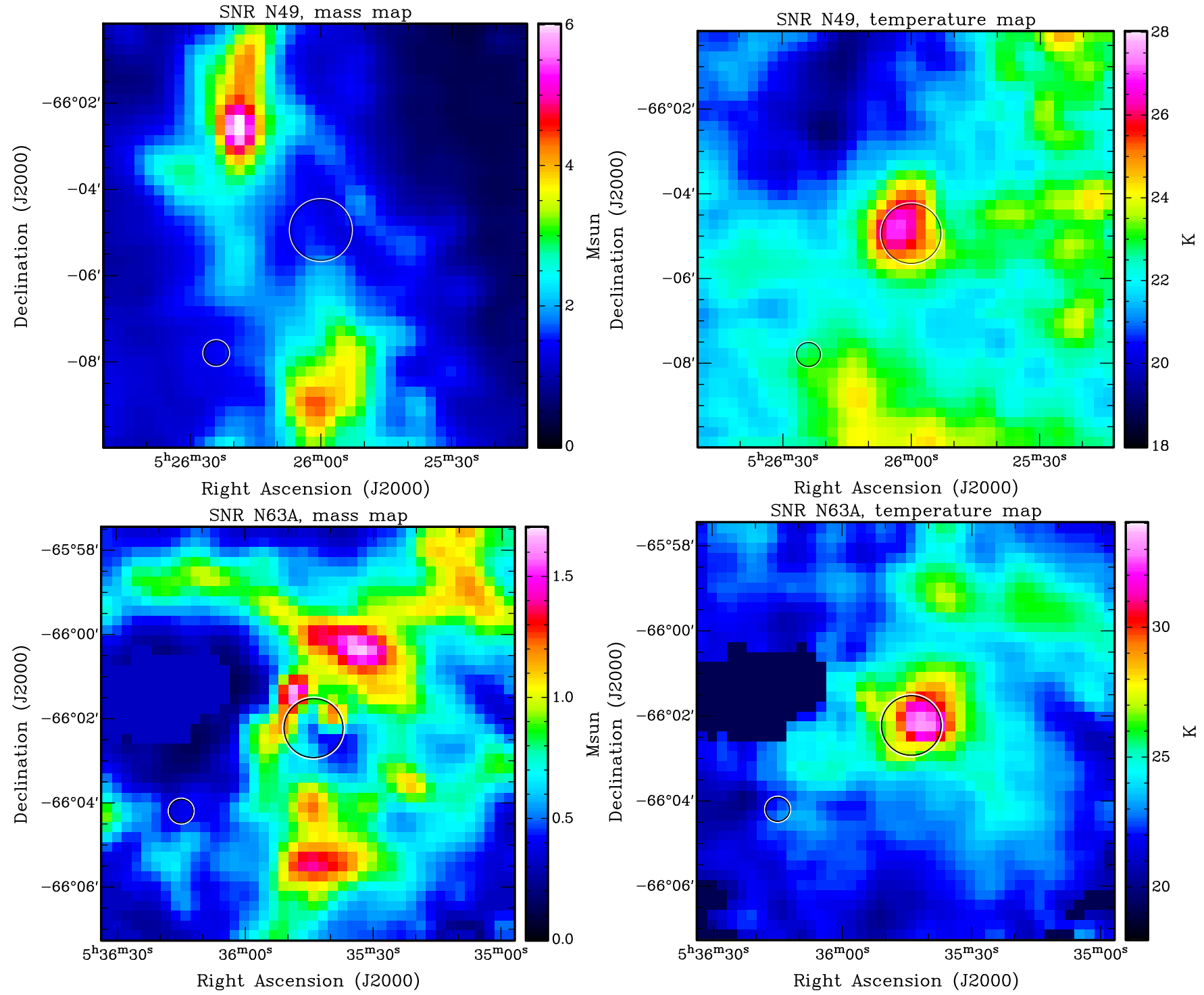

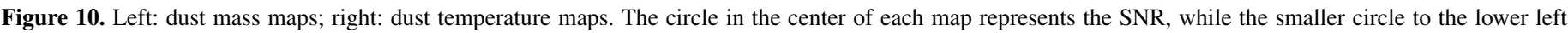
represents the beam size. Top: SNR N 49; bottom: SNR N 63A.

are of the order of the surface brightness uncertainties $(\sim 10 \%)$ and thus, our adopted model describes the data well. A more sophisticated model and error analysis can be found in Gordon et al. (2014), who used the same data but different $\kappa$ and $\beta$ as free parameters.

\subsection{Maps of Dust Mass and Temperature}

Here we show and describe the actual maps for a selection of SNRs, with the remainder presented in the Appendix. In Section 4, we will give our conclusions from these data interpreting the general lack of dust in the mass maps at the position of most of the SNRs by sputtering of the dust by shocks, and notice that the temperature is warmer in the direction of SNRs.

$$
\text { 3.4.1. } N 49
$$

The maps of mass and temperature of the dust in and around N49 are displayed in Figure 10, top. The progenitor has an estimated mass of $20 M_{\odot}$ (Hill et al. 1995); this is a remnant resulting from core collapse. Elevated temperatures are seen at the location of the "blob", an interstellar dust cloud which is heated by the SNR shock; this bright cloud is visible on the original Spitzer and Herschel images of the object (Williams et al. 2006; Otsuka et al. 2010). We conclude that we do not see this cloud so prominently because it is massive, but because it is heated.

\subsection{2. $N 63 A$}

The maps of mass and temperature in and around N 63A are displayed in Figure 10, bottom. The progenitor is likely to have been massive (Hughes et al. 1998), and there is a large H II region to its northwestern side. The warmest, northwestern part of the SNR corresponds to the shocked lobes, which have a high contribution from line emission at the $24 \mu \mathrm{m}$ flux (Caulet et al. 2012). We also notice the lack of dust in this SNR. The remnant is detected with Spitzer (Williams et al. 2006).

\subsubsection{N132D}

The maps of mass and temperature for N 132D are displayed in Figure 11, top. This is a young, O-rich SNR, thought to 

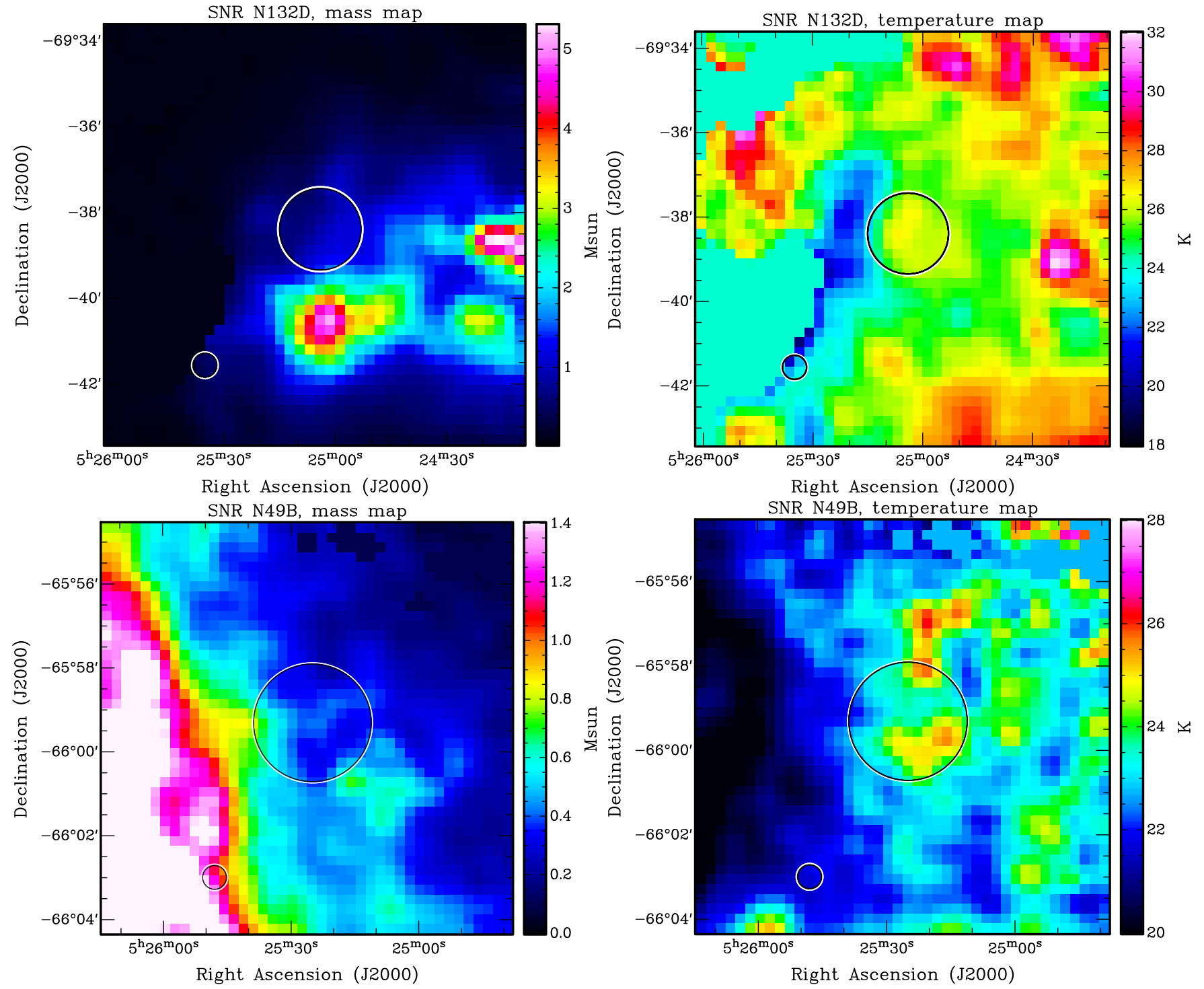

Figure 11. Like Figure 10, but for (top) SNR N 132D, and (bottom) SNR N 49B.

have a Ib progenitor (Vogt \& Dopita 2011). Rho et al. (2009) mapped IR spectral lines arising from the ejecta of this SNR. The remnant was detected with Spitzer (Williams et al. 2006). Tappe et al. (2006) reported the destruction of PAHs/grains in the supernova blast wave via thermal sputtering. N 132D has warmed up the little dust at the location of the SNR itself.

$$
\text { 3.4.4. } N 49 B
$$

This SNR was detected with Spitzer by Williams et al. (2006). The maps for N 49B are displayed in Figure 11, bottom. Despite its mature age of $\approx 10,000 \mathrm{yr}$, its influence through the heating and lack of dust is clear.

\subsubsection{DEM L71}

The maps for DEM L71 are displayed in Figure 12, top. This is a young type Ia remnant; $0.034 M_{\odot}$ of warm dust in this SNR was measured by Williams et al. (2010).

$$
\text { 3.4.6. } N 157 B
$$

For N 157B the maps are displayed in Figure 12, middle. This SNR, near to the Tarantula Nebula mini-starburst, has resulted from the core collapse of a massive progenitor, 20-25 $M_{\odot}$
(Micelotta et al. 2009, who detected it with Spitzer). It would be interesting to find out if the massive dust structure in the direction of this object is connected to the remnant.

\subsubsection{DEM L316A and DEM L316B}

These two objects are shown in Figure 12, bottom. There was an unresolved question whether these two objects are interacting and how close they are (Williams et al. 2005). These data suggest that they do belong to similar FIR environments, although the first one is of Ia and the second one of CC origin.

\subsubsection{Other SNRs of Note}

Many SNRs show signs of dust removal and/or heating. One of the "hidden" remnants, the Honeycomb is devoid of dust compared to its surroundings while lacking any signs of heating. Other SNRs showing signs of dust removal and/or heating within the SNR include 0453-68.5 (detected with Spitzer; Williams et al. 2006), N 4, the type Ia SNR 0519-69.0 (detected with Spitzer; Borkowski et al. 2006b), DEM L241, N 23, B 0548-70.4 (heated dust; detected with Spitzer; Borkowski et al. 2006b), SNR 0520-69.4 (probably removal and heating on the edges), DEM L204 devoid of dust, DEM L109 and a 

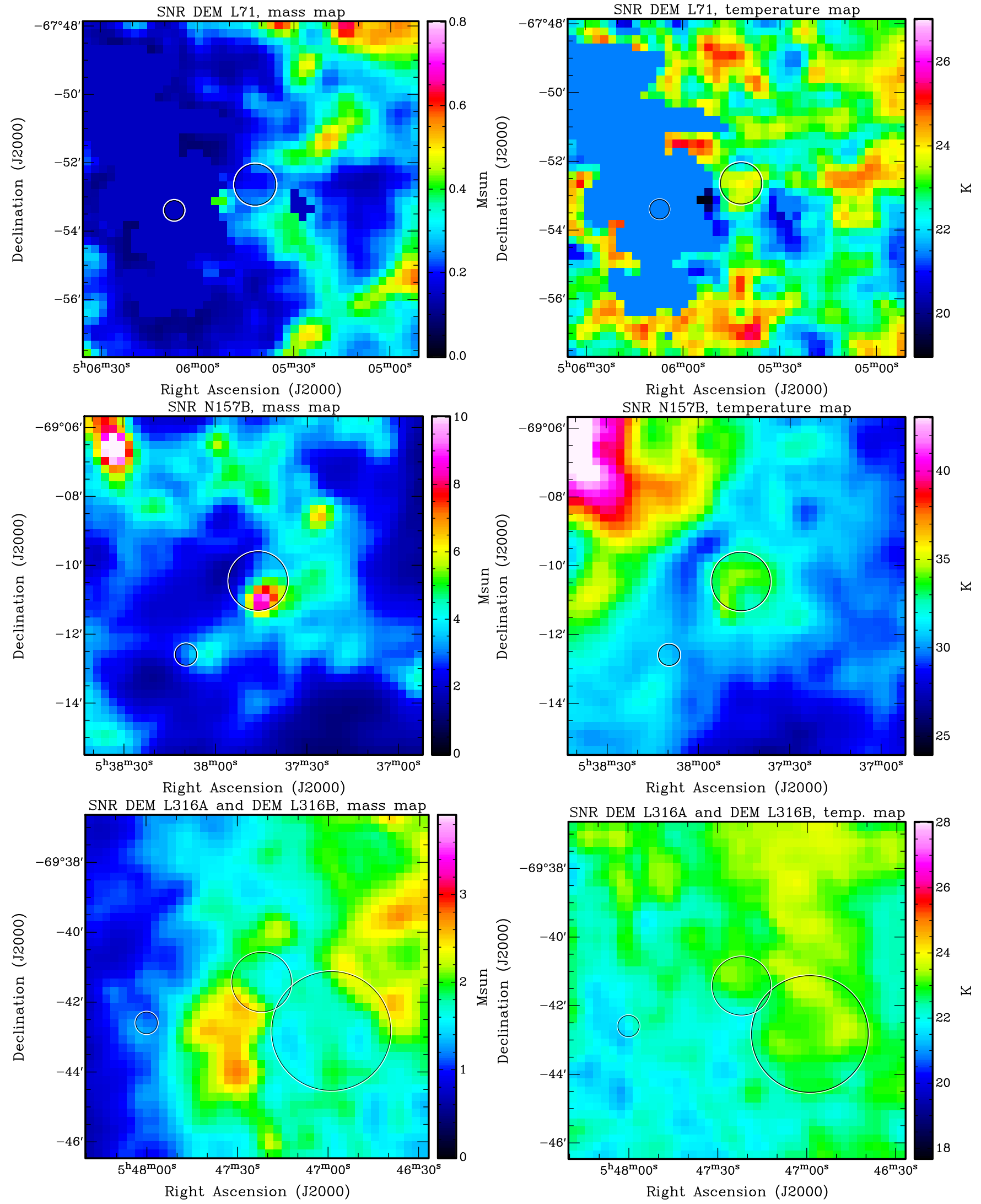

Figure 12. Like Figure 10, but for (top) SNR DEM L71, (middle) SNR N 157B, and (bottom) SNRs DEM L316A and DEM L316B. 
nearby compact SNR candidate, J 051327-6911 (Bojičić et al. 2007) appearing to heat a nearby dust cloud.

Several SNRs appear to interact with interstellar clouds at their periphery. Young N 158A (0540-69.3) (progenitor of 20-25 $M_{\odot}$, Williams et al. 2008) is possibly interacting with a dense cloud to the north. Williams et al. (2008) detected its PWN with Spitzer at wavelengths $\leqslant 24 \mu \mathrm{m}$, but did not find any IR detection of the shell. They found dust synthesized in the SNR, heated to $50-60 \mathrm{~K}$ by the shock wave generated by the PWN. The data we have only show possible heating of the surrounding medium, but not an obvious influence by this SNR. The young SNR N $103 \mathrm{~B}$ is not resolved; only $R_{70 / 24}$ shows some impact of this SNR. Other SNRs interacting (or about to interact) with surrounding clouds include N9, B 0507-7029, SNR 0450-709, and DEM L299.

Some SNRs are confused. DEM L218 (MCELS J 05307008), type Ia (de Horta et al. 2012), is covered with the object that Blair et al. (2006) called SNR 0530-70.1. N 159 (0540-697) is in the complicated region, close to the blackhole high-mass X-ray binary LMC X-1 and a bright $\mathrm{H}$ II region to the southwest (Seward et al. 2010). SN 1987A is utterly unresolved and barely visible on our mass map (we remind the reader that our maps are constructed at the angular resolution of the $500 \mu \mathrm{m}$ data).

\subsubsection{Pulsar Wind Nebulae}

Some SNRs contain a PWN, which could make a synchrotron contribution to the FIR spectrum or provide an additional mechanism to heat dust. The SNRs which have been connected with pulsars in the LMC are N 49 (Park et al. 2012), N 206, 0453-68.5, B 0540-693 in N 158A, N 157B, DEM L241 (Hayato et al. 2006), and DEM L214 (J 0529-6653; Bozzetto et al. 2012). On the other hand, while all PWNe are powered by pulsars, PWNe are known without a detected pulsar (Gaensler et al. 2003a), e.g., N 23 (Hayato et al. 2006). We did not find signatures of PWNe on any of our maps.

\section{DISCUSSION}

In Sections 4.1 and 4.2, we discuss the influence of the population of SNRs on the interstellar dust mass and temperature. In Section 4.3, we argue for a lack of evidence for large amounts of dust having formed in SNRs and survived. Given the extant literature which consistently indicates significant destruction of dust within SNRs by the reverse and forward shocks and hot gas (Barlow 1978; Jones et al. 1994; Borkowski et al. 2006b; Bianchi \& Schneider 2007; Nozawa et al. 2007; Nath et al. 2008; Silvia et al. 2010; Sankrit et al. 2010) we interpret the removal of dust as due to sputtering, although we cannot prove conclusively on the basis of our data that some dust may not be simply pushed out of the way. We attempt to quantify the amount of sputtered and/or pushed dust in Section 4.4, based on the difference of column density between SNR surroundings and SNRs. We end with a brief discussion about the thickness of the interstellar dust layer (Section 4.5).

\subsection{Influence of SNRs on Interstellar Dust Mass}

In Figure 13 (top panel), we present the radial profiles, the average dust column density in and around SNRs as a function of radial distance to the center of the SNR, for a sub-sample of 22 SNRs, where each successive bin comprises a $6.8 \mathrm{pc}$ wide annulus. The blue circles mark where the SNR radius ends. While often less dust is seen toward the SNR-although
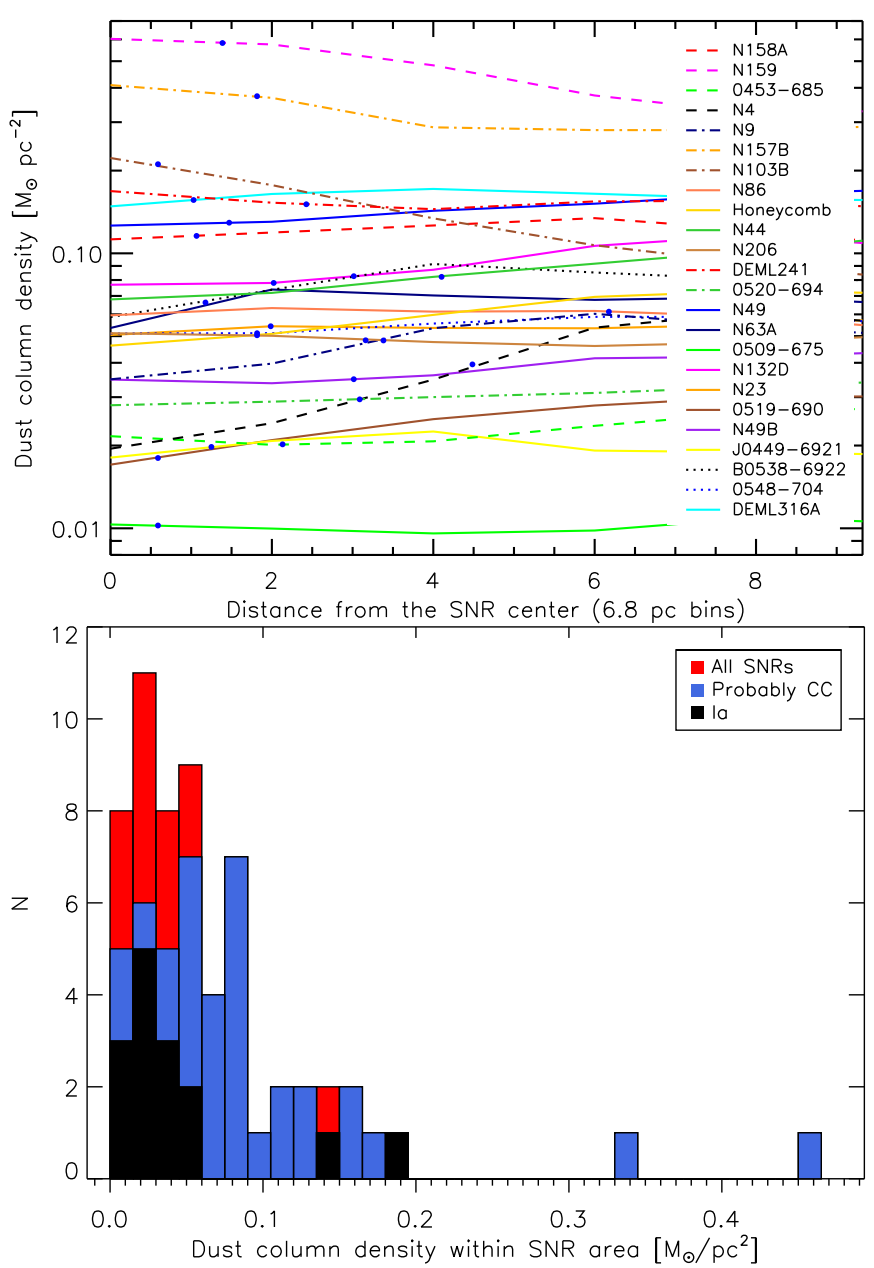

Figure 13. Top: radial profiles of dust mass distribution across SNRs and their environment. The blue circles mark the SNR radius. Bottom: histogram of the distribution of the average dust mass $\left[M_{\odot} \mathrm{pc}^{-2}\right]$ within one diameter of Ia, core-collapse, and all SNRs together.

sometimes the opposite is seen-the radial profiles are generally fairly flat; this indicates that the amount of (cold) dust that is removed is relatively small compared to the amount of dust in the LMC in that direction. These profiles also reflect a general relation between SN progenitor type and ISM density, as core-collapse $\mathrm{SNe}$ are usually seen in regions of recent star formation whereas SNe Ia often bear no memory of their natal environment. On this basis, N 157B and N 159 stand out in terms of ISM density; they are bright at FIR and radio wavelengths and their progenitors are probably massive.

To examine the difference in ISM surrounding CC and Ia SNRs, in the bottom panel of Figure 13 we show the distributions of average dust mass within the SNR diameter, separately for CC, Ia, and all SNRs together (for typing see Section 2.1). Ias are usually in dust-poor environments, but there are exceptions such as the probable prompt Ia SNRs DEM L316A and N 103B, that are seen in the direction of dense ISM. According to the $\mathrm{K}-\mathrm{S}$ test, there is a $1.6 \%$ chance that these two data sets come from the same distribution.

Some of the SNRs that we claim here to sputter and/or push away dust are already claimed to be dust destroyers based on Spitzer observations. With Herschel data the lack of dust can actually be observed for SNRs that have enough dust to interact with, since they enable us to see the colder dust that surrounds SNRs. 
If dust were pushed out of the way, we should see dust piling up around the rims of SNRs - which we do not see, but we might not be able to resolve it. For most of the remnants $(D \gtrsim 10 \mathrm{pc})$ the dust that formed in the progenitor envelope and CSM must have been significantly sputtered and/or pushed away by shocks in early stages. Only if the progenitor star was an early-type star could it have formed an interstellar bubble of a size $\sim 30 \mathrm{pc}$ (Castor et al. 1975), ${ }^{22}$ otherwise the dust around remnants is not directly connected with the CSM of the progenitor but with the pre-existing ISM dust, whose density may be related indirectly to the progenitor type.

As Forest et al. (1988) have shown, there seems to be a significant association between SNRs and H II regions, suggesting the preponderance of core-collapse SNRs in the LMC. The effect that they observed might also be attributed to SNRs being rendered visible by virtue of their interaction with the ISM (or CSM). At our dust mass maps many SNRs are close to, or at the rim of, dusty structures (clouds). That could hide the influence of SNRs to be visible for the observer in two ways: if the cloud is irregular and inhomogeneous, we would not see a lack of dust in SNRs; if the SNR is next to the cloud from our perspective, then it is more difficult to claim that it has removed any dust and not possible to estimate how much.

Also, large SNRs $(D>100 \mathrm{pc})$ subtend a larger area on the sky than the annulus, which tends to reduce the difference between inner and outer mass of the dust. On the other hand, the annuli of the smallest SNRs are more likely to be more massive than areas within remnants from the same reason.

\subsection{Influence of SNRs on Interstellar Dust Temperature}

Within SNRs there is usually a higher temperature-even for the older remnants. In older remnants the dust temperature often peaks near the edges or outside of the remnants, which can probably also be a sign that the dust has been removed. In Figure 14 (top), we show the ratio of inner and outer (from annuli 20 pc thick) temperature and, in Figure 14 (bottom), we show the temperatures for the individual SNRs (both derived from temperature maps while the errors are standard deviations) which indicates that most of the SNRs do heat up the surroundings and that the temperature of this dust is somewhat higher than the temperature of the interstellar radiation field. The heating of the dust is most prevalent in the more compact, presumably younger SNRs as well as the ones with a higher dust content.

The temperature maps generally show heating of dust on SNR locations, usually in SNRs that are detected with Spitzer, although here we use only Herschel data. Since the dust is being eroded and cooled, this heating is only seen in young and sufficiently dense SNRs.

We do not exclude the possibility that the heated dust belongs to the closer ISM surroundings of SNRs.

\subsection{Production of Dust in SNRs}

It is possible that all SNRs create some dust in their ejecta, but it is not possible to recognize with the maps we have. The youngest SNR in our sample is SN 1987A. It has produced $\sim 0.6 M_{\odot}$ of cold dust (see Matsuura et al. 2011; Lakićević et al. 2011; Indebetouw et al. 2014). It is unresolved, stands out on the Spitzer and short- $\lambda$ Herschel images but not at $500 \mu \mathrm{m}$. On our mass map the mass is slightly higher at the place of that

\footnotetext{
22 The winds of red supergiant SN progenitors are slow, $v \ll 100 \mathrm{~km} \mathrm{~s}^{-1}$, and
} such CSM is quickly over-run by the SNR (see, e.g., van Loon 2010).
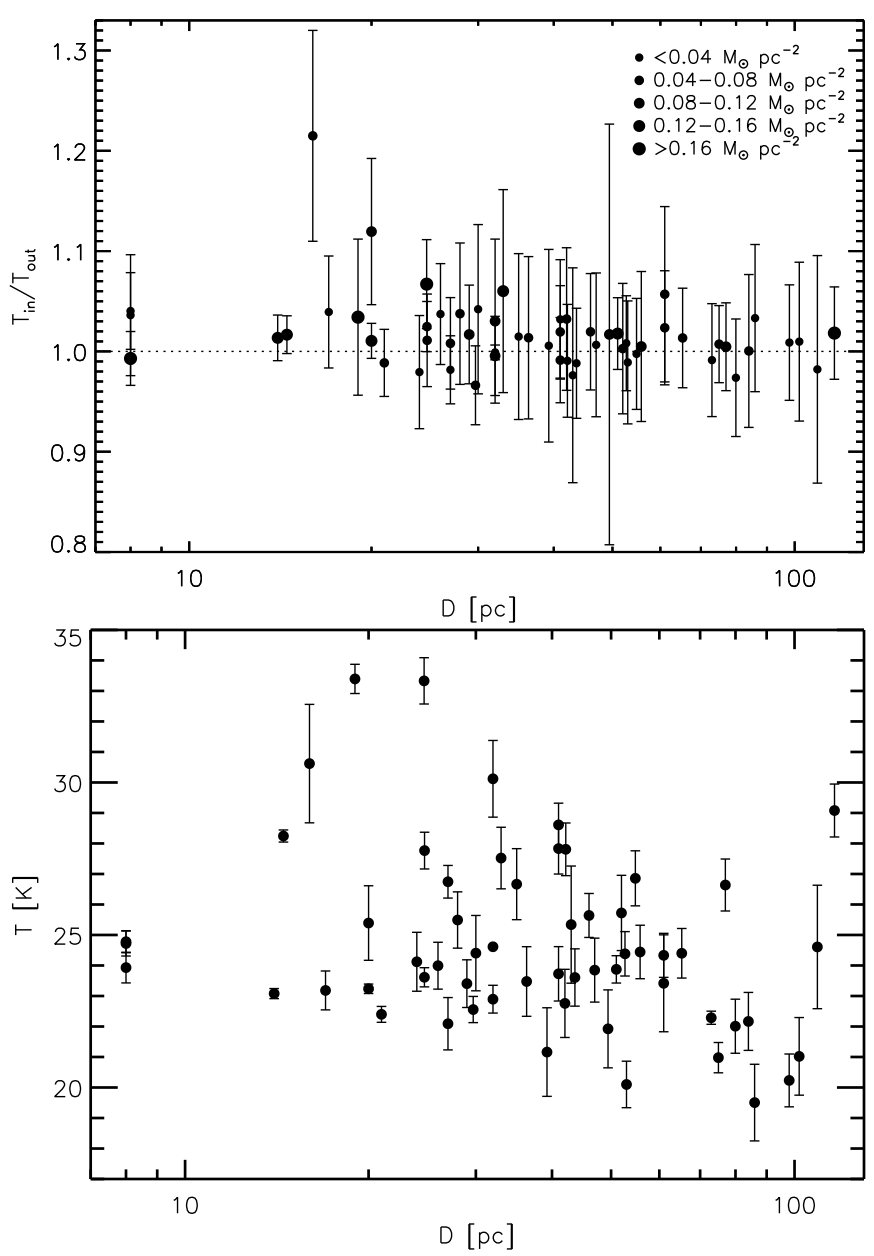

Figure 14. Top: ratio of average dust temperature within and outside the SNR vs. SNR diameter. The size of the symbol is proportional to the average dust column density in the direction of the SNR. Bottom: temperature within SNRs vs. diameters.

object, but this is barely visible-only because the surrounding ISM is not dense.

Another object that is probably in the free expansion phase (other objects are in later stages - most of the material associated with them is swept up ISM) is N 103B. N 103B might not be resolved on our maps; it has reduced $R_{70 / 24}$ compared to the surrounding ISM, but other ratios do not show that characteristic. It may indeed be in a rare environment, behind (or in front of) the massive cloud (Dickel et al. 1995). We see no dust production here distinguishable, but we do not expect it, since it is a Ia remnant.

Almost all other SNRs are larger than 50", and cover $>50 \mathrm{pc}^{2}$. Even if $1 M_{\odot}$ of dust were present in the ejecta, even if it is not spread on more than $\sim 3 \operatorname{arcsec}^{2}$, in an evolved SNR this would correspond to a surface density $<0.009 M_{\odot} \mathrm{pc}^{-2}$, i.e., often below that of the surrounding ISM $\left(\sim 0.01-0.6 M_{\odot} \mathrm{pc}^{-2}\right.$; Figure 13).

\subsection{How Much Dust Have SNRs Removed?}

We estimate the amount of dust that is removed by the SNRs by comparing the dust column density toward the SNR with that of within a $20 \mathrm{pc}$ thick annulus. Assuming that both reflect the ISM dust in those directions, the difference will correspond to the amount of dust that was removed (or added) by the SNR. In Table 4, we list the determinations for nearly all (60) SNRs. The average dust column density within the SNR is $N_{\text {in }}$ and within an 
Table 4

Name of SNR, Dust Column Densities within $\left(N_{\text {in }}\right)$ and outside $\left(N_{\text {out }}\right)$ the $\mathrm{SNR}$ in $M_{\odot} \mathrm{pc}^{-2}$, and the Total Amount of Dust That Could Be Removed by the SNR $\left(M\left[M_{\odot}\right]\right)$

\begin{tabular}{|c|c|c|c|}
\hline Name & $N_{\text {in }} \pm \Delta N_{\text {in }}$ & $N_{\text {out }} \pm \Delta N_{\text {out }}$ & $M \pm \Delta M$ \\
\hline B $0519-690$ & $0.015 \pm 0.004$ & $0.024 \pm 0.007$ & $0.9 \pm 0.3$ \\
\hline DEM L71 & $0.019 \pm 0.005$ & $0.021 \pm 0.006$ & $0.20 \pm 0.07$ \\
\hline B $0509-675$ & $0.009 \pm 0.003$ & $0.01 \pm 0.003$ & $0.04 \pm 0.02$ \\
\hline N 103B & $0.19 \pm 0.05$ & $0.13 \pm 0.04$ & $\cdots$ \\
\hline $0548-704$ & $0.05 \pm 0.02$ & $0.06 \pm 0.02$ & $4 \pm 2$ \\
\hline DEM L316A & $0.15 \pm 0.04$ & $0.16 \pm 0.05$ & $\cdots$ \\
\hline N9 & $0.05 \pm 0.02$ & $0.05 \pm 0.02$ & $\cdots$ \\
\hline $0534-699$ & $0.034 \pm 0.009$ & $0.033 \pm 0.009$ & $\cdots$ \\
\hline DEM L238 & $0.009 \pm 0.003$ & $0.01 \pm 0.003$ & $\cdots$ \\
\hline DEM L249 & $0.04 \pm 0.01$ & $0.031 \pm 0.008$ & $\cdots$ \\
\hline $0520-694$ & $0.027 \pm 0.007$ & $0.033 \pm 0.009$ & $11 \pm 3$ \\
\hline DEM L204 & $0.008 \pm 0.003$ & $0.011 \pm 0.003$ & $\cdots$ \\
\hline $0450-709$ & $0.031 \pm 0.008$ & $0.035 \pm 0.009$ & $\cdots$ \\
\hline HР99498 & $0.019 \pm 0.005$ & $0.017 \pm 0.005$ & $\ldots$ \\
\hline DEM L218 & $0.023 \pm 0.006$ & $0.018 \pm 0.005$ & $\cdots$ \\
\hline N 23 & $0.05 \pm 0.02$ & $0.06 \pm 0.02$ & $4 \pm 2$ \\
\hline N 132D & $0.08 \pm 0.02$ & $0.10 \pm 0.03$ & $\cdots$ \\
\hline N 157B & $0.34 \pm 0.09$ & $0.29 \pm 0.08$ & $\ldots$ \\
\hline $\mathrm{N} 44$ & $0.09 \pm 0.03$ & $0.11 \pm 0.03$ & $\cdots$ \\
\hline N 158A & $0.13 \pm 0.04$ & $0.13 \pm 0.04$ & $\ldots$ \\
\hline N 206 & $0.05 \pm 0.02$ & $0.05 \pm 0.02$ & $\cdots$ \\
\hline N 120 & $0.08 \pm 0.03$ & $0.10 \pm 0.03$ & $\cdots$ \\
\hline $\mathrm{N} 49 \mathrm{~B}$ & $0.035 \pm 0.009$ & $0.04 \pm 0.02$ & $12 \pm 4$ \\
\hline N 49 & $0.12 \pm 0.04$ & $0.14 \pm 0.04$ & $6 \pm 2$ \\
\hline N 11L & $0.07 \pm 0.02$ & $0.08 \pm 0.03$ & $4 \pm 2$ \\
\hline N 86 & $0.06 \pm 0.02$ & $0.04 \pm 0.02$ & $\cdots$ \\
\hline $0453-685$ & $0.019 \pm 0.005$ & $0.024 \pm 0.007$ & $\cdots$ \\
\hline N 63A & $0.06 \pm 0.02$ & $0.07 \pm 0.02$ & $\ldots$ \\
\hline DEM L203 & $0.07 \pm 0.02$ & $0.08 \pm 0.02$ & $\cdots$ \\
\hline DEM L241 & $0.15 \pm 0.04$ & $0.15 \pm 0.04$ & $\cdots$ \\
\hline DEM L299 & $0.09 \pm 0.03$ & $0.10 \pm 0.03$ & $\cdots$ \\
\hline DEM L109 & $0.08 \pm 0.03$ & $0.07 \pm 0.02$ & $\cdots$ \\
\hline MCELS J0506-6541 & $0.025 \pm 0.007$ & $0.026 \pm 0.007$ & $\cdots$ \\
\hline $0507-7029$ & $0.031 \pm 0.008$ & $0.04 \pm 0.01$ & $\cdots$ \\
\hline $0528-692$ & $0.012 \pm 0.004$ & $0.013 \pm 0.004$ & $2.0 \pm 0.8$ \\
\hline DEM L214 & $0.003 \pm 0.001$ & $0.003 \pm 0.001$ & $\cdots$ \\
\hline $0532-675$ & $0.07 \pm 0.02$ & $0.07 \pm 0.02$ & $\ldots$ \\
\hline Honeycomb & $0.05 \pm 0.02$ & $0.06 \pm 0.02$ & $10 \pm 3$ \\
\hline $0536-6914$ & $0.17 \pm 0.05$ & $0.15 \pm 0.04$ & $\cdots$ \\
\hline DEM L256 & $0.09 \pm 0.03$ & $0.08 \pm 0.02$ & $\ldots$ \\
\hline N 159 & $0.5 \pm 0.2$ & $0.4 \pm 0.2$ & $\cdots$ \\
\hline DEM L316B & $0.15 \pm 0.04$ & $0.16 \pm 0.05$ & $\cdots$ \\
\hline J0550-6823 & $0.05 \pm 0.02$ & $0.05 \pm 0.02$ & $\cdots$ \\
\hline В $0450-6927$ & $0.14 \pm 0.04$ & $0.11 \pm 0.03$ & $\cdots$ \\
\hline $0454-7005$ & $0.012 \pm 0.004$ & $0.014 \pm 0.004$ & $\cdots$ \\
\hline DEM L214 & $0.009 \pm 0.003$ & $0.011 \pm 0.003$ & $\cdots$ \\
\hline MCELS J04496921 & $0.12 \pm 0.03$ & $0.10 \pm 0.03$ & $\cdots$ \\
\hline N 186D & $0.05 \pm 0.02$ & $0.05 \pm 0.02$ & $\cdots$ \\
\hline $0521-6542$ & $0.04 \pm 0.01$ & $0.04 \pm 0.02$ & $\cdots$ \\
\hline MCELS J0448-6658 & $0.018 \pm 0.005$ & $0.020 \pm 0.006$ & $3.0 \pm 0.8$ \\
\hline $\mathrm{N} 4$ & $0.05 \pm 0.02$ & $0.06 \pm 0.02$ & $\cdots$ \\
\hline RXJ0507-68 & $0.025 \pm 0.007$ & $0.028 \pm 0.008$ & $\cdots$ \\
\hline В $0528-7038$ & $0.010 \pm 0.003$ & $0.017 \pm 0.005$ & $\ldots$ \\
\hline $0538-693$ & $0.08 \pm 0.03$ & $0.08 \pm 0.02$ & $\cdots$ \\
\hline $0538-6922$ & $0.08 \pm 0.03$ & $0.08 \pm 0.03$ & $\cdots$ \\
\hline В $0449-693$ & $0.11 \pm 0.03$ & $0.12 \pm 0.03$ & $10 \pm 3$ \\
\hline J0508-6830 & $0.04 \pm 0.02$ & $0.08 \pm 0.03$ & $27 \pm 7$ \\
\hline J0511-6759 & $0.023 \pm 0.006$ & $0.028 \pm 0.008$ & $3.0 \pm 0.8$ \\
\hline J0514-6840 & $0.022 \pm 0.006$ & $0.021 \pm 0.006$ & $\cdots$ \\
\hline J0517-6759 & $0.05 \pm 0.02$ & $0.06 \pm 0.02$ & $\cdots$ \\
\hline
\end{tabular}

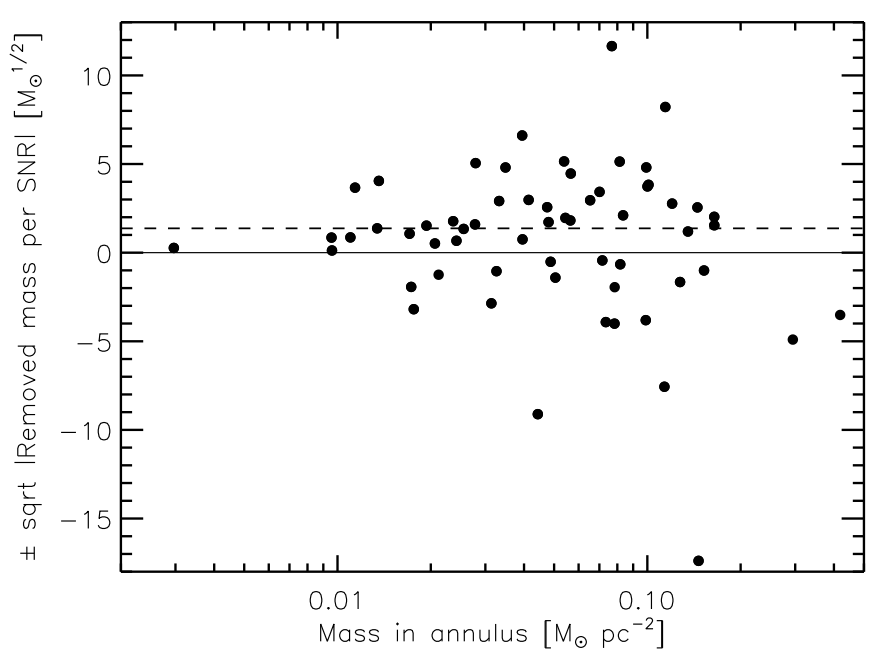

Figure 15. Removed dust mass vs. dust mass in an annulus surrounding the SNR. Square-root values are plotted to limit the dynamical range, with negative masses $(M<0)$ represented by $-\sqrt{-M}$. The median value is indicated by the dashed line.

annulus surrounding the SNR is $N_{\text {out }}$. We only quote the values for the removed dust mass $M$ for those cases we are reasonably confident about (for these SNRs we believe that they did remove dust and that the difference in dust mass is not caused by the accidental position of the SNR next to the cloud). We find that the latter (15 SNRs) show a wide range in dust removal, but with a mean of $6.5 M_{\odot}$ (and a median of $\sim 4 M_{\odot}$ ) well above the typically inferred amounts of dust that are produced in the ejecta $\left(<1 M_{\odot}\right)$. If this is representative of the SNR population as a whole we would obtain $M_{1} \sim 390 M_{\odot}$. If instead of the mean, we use the median value of these 15 SNRs, we will have $M_{1}^{\prime}=240 M_{\odot}$. In Table 4, the errors for $N_{\text {in }}$ and $N_{\text {out }}$ include the uncertainties of the fitting as well as the uncertainty of $\kappa$ which is $\sim 25 \%$ (Gordon et al. 2014). The uncertainties of $M$ are found by adding in quadrature the combined uncertainties of the fitting of $N_{\text {in }}$ and $N_{\text {out }}$ together with the uncertainty of $\kappa$ and multiplying with the areas of the objects.

Because this may be biased, we attempt to estimate the result for the entire sample of SNRs, including negative values, as follows.

$$
M_{2}=\sum_{i=1}^{N}\left[\pi(D / 2)^{2} \times\left(N_{\text {out }}-N_{\text {in }}\right)\right],
$$

where $D$ is the SNR diameter (in parsecs), and the sum is over $N$ objects. Using this approach we obtain $M_{2}=-13.6 M_{\odot}$ of dust removed by $N=60$ SNRs in the LMC. This result is driven by a few severe outliers for which the individual estimates are particularly uncertain (Figure 15), yet 40 out of 60 SNRs have less dust within their diameter than in the annulus.

Our third estimate is based on the median rather than the sum (or average):

$$
M_{2}^{\prime}=60 \times \operatorname{Median}_{i=1}^{N}\left[\pi(D / 2)^{2} \times\left(N_{\text {out }}-N_{\text {in }}\right)\right] .
$$

Now, we obtain $M_{2}^{\prime} \sim 113 M_{\odot}$. This, on the other hand, may exclude rare, but real, more prominent contributors to dust removal.

An alternative way of estimating the combined effect of the SNR population within the $\mathrm{LMC}$ is based on an empirical Monte Carlo simulation. We generate 10,000 sets of values for mass $(m)$ and for diameter $(D)$. These are drawn from the positive domain 

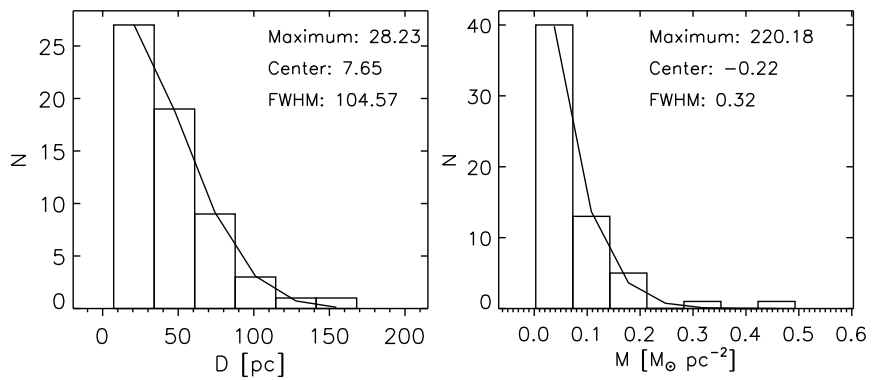

Figure 16. Left: distribution of the diameters of the SNRs in our sample. Right: distribution of the mass within the SNRs in our sample.

of a Gaussian, where the width is set by $\sigma=0.13 M_{\odot} \mathrm{pc}^{-2}$ for $m$ and $\sigma=44.4 \mathrm{pc}$ for $D$ (Figure 16). For each of these pairs of values $(m, D)$ we derive the removed mass, from which we obtain the average removed mass per SNR and thence

$$
M_{3}=60 \times\left\langle\pi(D / 2)^{2} \times m \times 0.09 \times \frac{40}{60}\right\rangle,
$$

where $40 / 60$ is the probability that the SNR is removing the dust that we see in its direction-40 is the number of SNRs with $\left(N_{\text {out }} / N_{\text {in }}\right)>1$, in our sample of 60 SNRs; 0.09 is the average fraction of removed dust mass as compared to the dust mass that is seen in the direction of the SNR $\left(\left\langle N_{\text {out }} / N_{\text {in }}\right\rangle=1.09\right)$. We thus obtain $M_{3} \sim 389 M_{\odot}$. The results of these five estimates are given in Table 5 .

While extrapolation of the 'cleanest sample', $\mathrm{M}_{1}$ and Monte Carlo give high values, $\mathrm{M}_{2}$ is too influenced by the fore/ background of three to four very massive SNRs that have $N_{\text {out }}<N_{\text {in }}$. We will adopt the average of the values in Table 5, i.e., $224 M_{\odot}$ removed by the whole sample.

The different approaches that we followed in the estimation of the removed dust give a range of a factor of \pm 2 , but the uncertainty of $\kappa$ causes also a similar factor (we compared $\kappa$ from this work with the one from Gordon et al. 2014 and found that our dust masses are $\sim 60 \%$ of the masses for their SMBB model due to $\kappa$ uncertainty). Therefore, our estimate of the total error of removed mass is a factor of three.

For an SN rate in the LMC of $d N / d t=10^{-2} \mathrm{yr}^{-1}$ (Filipović et al. 1998) and a mean lifetime $\tau_{\mathrm{SNR}}=10^{4} \mathrm{yr}$ for SNRs to be visible (Van den Bergh et al. 2004), the number of SNRs existing in the LMC should be $N=\tau_{\mathrm{SNR}} \times d N / d t=100$, i.e., double the sample considered here. Correcting for this, we deduce a total mass of removed dust of $M=373 M_{\odot}$, within the range $124-1119 M_{\odot}$. Thus, under the assumption that the dust is sputtered, we derive a dust destruction rate by SNRs in the LMC of $d M / d t=M / \tau_{\mathrm{SNR}}=0.037 M_{\odot} \mathrm{yr}^{-1}$ within a range of $0.012-0.11 M_{\odot} \mathrm{yr}^{-1}$. For a total interstellar dust mass in the LMC of $M_{\text {dust }}=7.3 \times 10^{5} M_{\odot}$ (Gordon et al. 2014) this would imply an interstellar dust lifetime of $\tau_{\text {dust }}=M_{\text {dust }} /(d M / d t) \sim 2 \times 10^{7} \mathrm{yr}$ within a range 0.7-6 $\times 10^{7} \mathrm{yr}$. Of course, not all of the interstellar dust is affected by SNRs to the same degree, and some dust may survive a lot longer.

\subsection{Thickness of the Dust Layer in the LMC}

We can use the $N_{\text {out }} / N_{\text {in }}$ values from Table 4 to estimate the thickness, $d$, of the dust layer within the LMC - and hence the average density - if we assume that all dust has been removed from within the SNRs. In that case, and assuming SNRs are spherical with diameter $D$ and fully embedded within the dust
Table 5

Mass Removed by SNRs in the LMC According to Various Methods

\begin{tabular}{llc}
\hline \hline ID & \multicolumn{1}{c}{ Method } & Removed Mass $M_{\odot}$ \\
\hline$M_{1}$ & Mean, good & 390 \\
$M_{1}^{\prime}$ & Median, good & 240 \\
$M_{2}$ & Mean, all & -13 \\
$M_{2}^{\prime}$ & Median, all & 113 \\
$M_{3}$ & Monte Carlo & 389 \\
\hline
\end{tabular}

layer, the volume in a column with area $A=\pi(D / 2)^{2}$ that contains dust is $A d$ outside the SNR and $A d-\frac{4}{3} \pi(D / 2)^{3}$ in the direction of the SNR. Dividing by $A$, we obtain column lengths of $d$ and $d-\frac{2}{3} D$, respectively. Assuming a constant density, then the surface mass densities would compare as $N_{\text {in }} / N_{\text {out }}=1-\frac{2 D}{3 d}$ and hence we could estimate a thickness (in pc)

$$
d=\frac{2}{3} D\left(1-\frac{N_{\text {in }}}{N_{\text {out }}}\right)^{-1},
$$

and average dust volume density $\rho=N_{\text {out }} / d$ in $M_{\odot} \mathrm{pc}^{-3}$.

We thus obtain a median value for the thickness of the dust layer within the LMC to be $d \sim 107 \mathrm{pc}$.

\section{CONCLUSIONS}

We present the first FIR and submillimeter analysis of the population of 61 SNRs in the LMC, based on Herschel images from the HERITAGE survey at 100, 160, 250, 350, and $500 \mu \mathrm{m}$ in combination with Spitzer 24 and $70 \mu \mathrm{m}$ images. These Herschel data allow us to estimate the mass of the cold interstellar dust. To that aim, we produce maps of dust mass and temperature. We reach the following conclusions.

1. Although the FIR surface brightness of SNRs is very similar to the one of the ISM, it is slowly decreasing with time, meaning that SNRs cool down and/or dust is removed from them, or the dust is sputtered. The radio surface brightness is weakly correlated with that of the FIR.

2. There is no evidence for large amounts of dust having formed and survived in SNRs. In fact, most of the dust seen in our maps is pre-existing.

3. If SNRs are "empty" in terms of dust, then we estimate a typical thickness of the ISM dust layer within the LMC of $\sim 107$ pc.

4. The ISM is generally denser around core-collapse SNRs than type Ia, but significant variations are seen between individual SNRs of either type.

5. We argue that SNRs sputter and heat cold interstellar dust by their hot plasma and shocks, which is evident from the dust and temperature maps. The data presented here do not, however, exclude the possibility that dust is pushed out of the sightline.

6. The amount of removed dust for all SNRs in the LMC is estimated to be $\sim 373_{-249}^{+746} M_{\odot}\left(3.7_{-2.5}^{+7.5} M_{\odot}\right.$ per SNR $)$. Under the assumption that all of that dust is sputtered, we derive a dust destruction rate of $0.037_{-0.025}^{+0.075} M_{\odot} \mathrm{yr}^{-1}$ and thus a lifetime of interstellar dust in the regions close to SNRs of $2_{-1.3}^{+4.0} \times 10^{7} \mathrm{yr}$.

We thank the referee for her/his constructive report and Dr. Eli Dwek for helpful advice. M.L. acknowledges an ESO/ Keele studentship. D.U. acknowledges support from the Ministry of Education, Science and Technological Development of the Republic of Serbia through project No. 176005. 

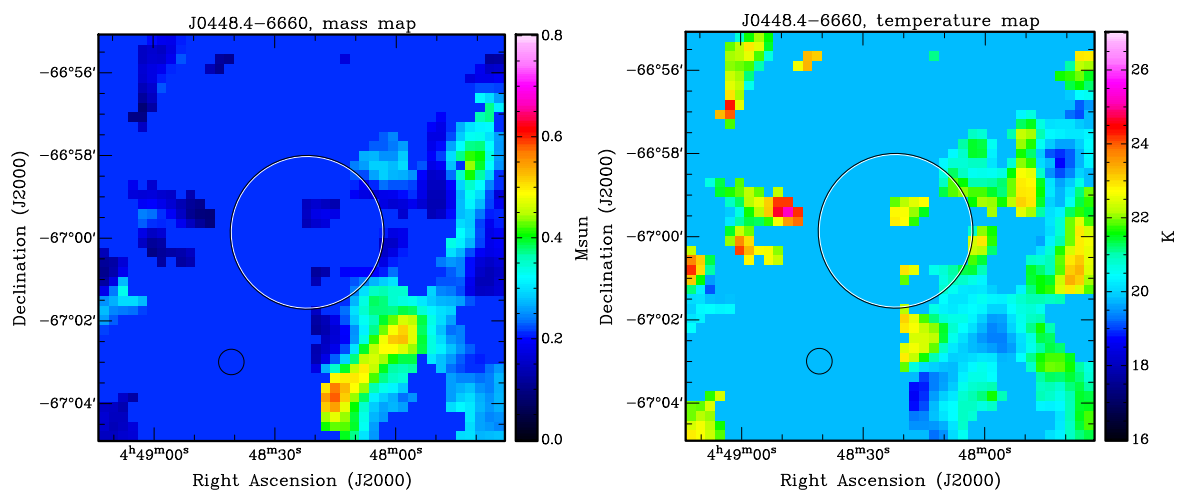

Figure 17. Dust mass (left) and dust temperature (right) maps of J 04486658.

(A complete figure set (50 images) is available.)

\section{APPENDIX}

\section{FIR ATLAS OF SNRs IN THE LARGE MAGELLANIC CLOUDS: MAPS OF DUST MASS AND TEMPERATURE}

In the online supporting material (see Figure 17), we present the maps of dust mass and temperature for the remaining SNRs from this paper. For each remnant, on the left side is the mass map and on the right side the temperature map (see Figure 17.1). The main conclusion based on these maps is that the SNRs remove and heat up the dust, since we notice that often there is less dust seen toward SNRs than in their surroundings and that the dust within SNRs is often warmer.

\section{REFERENCES}

Andersen, M., Rho, J., Reach, W. T., Hewitt, J. W., \& Bernard, J. P. 2011, ApJ, 742,7

Arbutina, B., Urošević, D., Stanković, M., \& Tešić, Lj. 2004, MNRAS, 350,346

Arendt, R. G., Dwek, E., Blair, W. P., et al. 2010, ApJ, 725, 585

Badenes, C., Harris, J., Zaritsky, D., \& Prieto, J. L. 2009, ApJ, 700, 727

Badenes, C., Maoz, D., \& Draine, B. T. 2010, MNRAS, 407, 1301

Barlow, M. J. 1978, MNRAS, 183, 367

Barlow, M. J., Krause, O., Swinyard, B. M., et al. 2010, A\&A, 518, L138

Bianchi, S., \& Schneider, R. 2007, MNRAS, 378, 973

Blair, W. P., Ghavamian, P., Sankrit, R., \& Danforth, C. W. 2006, ApJS, 165,480

Blair, W. P., Sankrit, R., \& Raymond, J. C. 2005, AJ, 129, 2268

Bojičić, I. S., Filipović, M. D., Parker, Q. A., et al. 2007, MNRAS, 378, 1237

Borkowski, K. J., Hendrick, S. P., \& Reynolds, S. P. 2006a, ApJ, 652, 1259

Borkowski, K. J., Williams, B. J., Reynolds, S. P., et al. 2006b, ApJL, 642, L141

Boyer, M. L., Srinivasan, S., Riebel, D., et al. 2012, ApJ, 748, 40

Bozzetto, L. M., Filipović, M. D., Crawford, E. J., et al. 2012, MNRAS, 420,2588

Casoli, F., \& Lequeux, J. 1998, Infrared Space Astronomy, Today and Tomorrow (Verlag: Springer)

Castor, J., McCray, R., \& Weaver, R. 1975, ApJL, 200, L107

Caulet, A., \& Williams, R. M. 2012, ApJ, 761, 107

Chu, Y., \& Kennicutt, R. C. 1988, AJ, 96, 1874

Chu, Y., Low, M.-M. M., García-Segura, G., Wakker, B., \& Kennicutt, R. C. 1993, ApJ, 414, 213

Compiègne, M., Verstraete, L., Jones, A., et al. 2011, A\&A, 525, A103

de Horta, A. Y., Filipović, M. D., Bozzetto, L. M., et al. 2012, A\&A, 540, A25

Desai, K. M., Chu, Y.-H., \& Gruendl, R. A. 2010, AJ, 140, 584

Dickel, J. R., \& Milne, D. K. 1995, AJ, 109, 200

Dwek, E., Galliano, F., \& Jones, A. P. 2007, ApJ, 662, 927

Elmhamdi, A., Danziger, I. J., Chugai, N., et al. 2003, MNRAS, 338, 939

Filipović, M. D., Pietsch, W., Haynes, R. F., et al. 1998, A\&AS, 127, 119

Forest, T., Spenny, D. L., \& Johnson, W. 1988, PASP, 100, 683

Fox, O., Skrutskie, M. F., Chevalier, R. A., et al. 2009, ApJ, 691, 650

Gaensler, B. M., Hendrick, S. P., Reynolds, S. P., \& Borkowski, K. J. 2003, ApJL, 594, L111
Gall, C. PhD thesis, Univ. Copenhagen

Gomez, H. L., Clark, C. J. R., Nozawa, T., et al. 2012a, MNRAS, 420, 3557

Gomez, H. L., Krause, O., Barlow, M. J., et al. 2012b, ApJ, 760, 96

Gordon, K. D., Roman-Duval, J., Bot, C., et al. 2014, ApJ, 797, 85

Haberl, F., Filipović, M. D., Bozzetto, L. M., et al. 2012, A\&A, 543, A154

Hayato, A., Bamba, A., Tamagawa, T., \& Kawabata, K. 2006, ApJ, 653, 280

Hendrick, S. P., Borkowski, K. J., \& Reynolds, S. P. 2003, ApJ, 593, 370

Hill, R. S., Cheng, K.-P., Bohlin, R. C., et al. 1995, ApJ, 446, 622

Hughes, J. P., Hayashi, I., \& Koyama, K. 1998, ApJ, 505, 732

Indebetouw, R., Matsuura, M., Dwek, E., et al. 2014, ApJL, 782, L2

Jones, A. P., Tielens, A. G. G. M., Hollenbach, D. J., \& McKee, C. F. 1994, ApJ, 433, 797

Lakićević, M., van Loon, J. Th., Patat, F., Staveley-Smith, L., \& Zanardo, G. 2011, A\&A, 532, L8

Lakićević, M., van Loon, J. Th., Stanke, T., De Breuck, C., \& Patat, F. 2012a, A\&A, 541, L1

Lakićević, M., Zanardo, G., van Loon, J. Th., et al. 2012b, A\&A, 541, L2

Lewis, K. T., Burrows, D. N., Hughes, J. P., et al. 2003, AJ, 582, 770

Maggi, P., Haberl, F., Kavanagh, P. J., et al. 2014, A\&A, 561, A76

Markwardt, C. B. 2009, in ASP Conf. Ser. 411, Astronomical Data Analysis Software and Systems XVIII, ed. D. A. Bohlender, P. Dowler, \& D. Durand (San Francisco, CA: ASP), 251

Matsuura, M., Dwek, E., Meixner, M., et al. 2011, Sci, 333, 1258

Meixner, M., Gordon, K. D., Indebetouw, R., et al. 2006, AJ, 132, 2268

Meixner, M., Panuzzo, P., Roman-Duval, J., et al. 2013, AJ, 146, 62

Mennella, V., Brucato, J. R., Colangeli, L., et al. 1998, ApJ, 496, 1058

Micelotta, E. R., Brandl, B. R., \& Israel, F. P. 2009, A\&A, 500, 807

Micelotta, E. R., Jones, A. P., \& Tielens, A. G. G. M. 2010, A\&A, 510, A36

Nath, B. B., Laskar, T., \& Shull, M. 2008, ApJ, 682, 1055

Nozawa, T., Kozasa, T., \& Habe, A. 2007, ApJ, 666, 955

Nozawa, T., Kozasa, T., Tominaga, N., et al. 2010, ApJ, 713, 356

Otsuka, M., van Loon, J. Th., Long, K. S., et al. 2010, A\&A, 518, L139

Park, S., Burrows, D. N., Garmire, G. P., \& Nousek, J. A. 2003a, ApJ, 586, 210

Park, S., Hughes, J. P., Slane, P. O., et al. 2003b, ApJL, 592, L41

Park, S., Hughes, J. P., Slane, P. O., et al. 2012, ApJ, 748, 117

Payne, J. L., White, G. L., \& Filipović, M. D. 2008, MNRAS, 383, 1175

Planck Collaboration, Ade, P. A. R., Alves, M. I. R., et al. 2014, A\&A, arXiv: 1405.0874

Reach, W. T., Rho, J., Tappe, et al. 2006, AJ, 131, 1479

Rest, A., Suntzeff, N. B., Olsen, K., et al. 2005, Natur, 438, 1132

Rho, J., Reach, W. T., Tappe, A., et al. 2009, in ASP Conf. Ser. 414, Cosmic Dust, Near and Far, ed. T. Henning, E. Grün, \& J. Steinacker (San Francisco, CA: ASP), 22

Rosado, M., Laval, A., Coarer, E. L., et al. 1993, A\&A, 272, 541

Rowlands, K., Gomez, H. L., Dunne, et al. 2014, MNRAS, 441, 1040

Sandstrom, K. M., Bolatto, A. D., Stanimirović, S., van Loon, J. Th., \& Smith, J. D. T. 2009, ApJ, 696, 2138

Sankrit, R., Williams, B. J., Borkowski, K. J., et al. 2010, ApJ, 712, 1092

Seok, J. Y., Koo, B.-C., Onaka, T., et al. 2008, PASJ, 60, S453

Seok, J. Y., Koo, B.-C., Onaka, T., et al. 2014, ApJ, 779, 134

Seward, F. D., Charles, P. A., Foster, D. L., et al. 2012, ApJ, 759, 123

Seward, F. D., Williams, R. M., Chu, Y.-H., Gruendl, R. A., \& Dickel, J. R. 2010, AJ, 140, 177

Seward, F. D., Williams, R. M., Chu, Y.-H., et al. 2006, ApJ, 640, 327

Silvia, D. W., Smith, B. D., \& Shull, J. M. 2010, ApJ, 715, 1575 
Someya, K., Bamba, A., \& Ishida, M. 2010, PASJ, 62, 1301

Stanimirović, S., Bolatto, A. D., Sandstrom, K., et al. 2005, ApJL, 632, L103

Szalai, T., \& Vinkó, J. 2013, A\&A, 549, A79

Tappe, A., Rho, J., \& Reach, W. T. 2006, ApJ, 653, 267

Temim, T., \& Dwek, E. 2013, ApJ, 774, 8

Temim, T., Sonneborn, G., Dwek, E., et al. 2012, ApJ, 753, 72

Urošević, D. 2003, Ap\&SS, 283, 75

Van den Bergh, S. 2004, The Galaxies of the Local Group (Cambridge: Cambridge Univ. Press)

van Loon, J. Th. 2010, in ASP Conf. Ser. 425, Hot and Cool: Bridging Gaps in Massive-star Evolution ed. C. Leitherer, P. Bennett, P. Morris, \& J. van Loon (San Francisco, CA: ASP), 279

van Loon, J. Th., Oliveira, J. M., Gordon, K. D., et al. 2010, AJ, 139, 68

Vogt, F., \& Dopita, M. A. 2011, Ap\&SS, 331, 521
Vukotić, B., Urošević, D., Filipović, M. D., \& Payne, J. L. 2009, A\&A, 503,855

Walker, A. R. 2012, Ap\&SS, 341, 43

Wang, Q. D., Gotthelf, E. V., Chu, Y.-H., \& Dickel, J. R. 2001, ApJ, 559, 275

Williams, B. PhD thesis, North Carolina State Univ. (arXiv:1005.1296)

Williams, B. J., Borkowski, K. J., \& Reynolds, S. P. 2008, ApJ, 687, 1054

Williams, B. J., Borkowski, K. J., Reynolds, S. P., et al. 2006, ApJL, 652, L33

Williams, R. M., \& Chu, Y.-H. 2005, ApJ, 635, 1077

Williams, R. M., Chu, Y.-H., Dickel, J. R., \& Gruendl, R. A. 2005, ApJ, 628,704

Williams, R. M., Chu, Y.-H., Dickel, J. R., et al. 1999, ApJ, 514, 798

Williams, R. M., Chu, Y.-H., Dickel, J. R., et al. 2004, ApJ, 613, 948

Williams, R. M., Chu, Y.-H., \& Gruendl, R. 2006, AJ, 132, 1877

Zanardo, G., Staveley-Smith, L., Ball, L., et al. 2010, ApJ, 710, 1515

Zhukovska, S. 2014, A\&A, 562, A76 